$1-1-1927$

\title{
Some Phases of the Relation of Temperature to the Development of Insects
}

Leonard Marion Peairs

Follow this and additional works at: https://researchrepository.wvu.edu/ wv_agricultural_and_forestry_experiment_station_bulletins

\section{Digital Commons Citation}

Peairs, Leonard Marion, "Some Phases of the Relation of Temperature to the Development of Insects" (1927). West Virginia Agricultural and Forestry Experiment Station Bulletins. 208.

https://researchrepository.wvu.edu/wv_agricultural_and_forestry_experiment_station_bulletins/208 


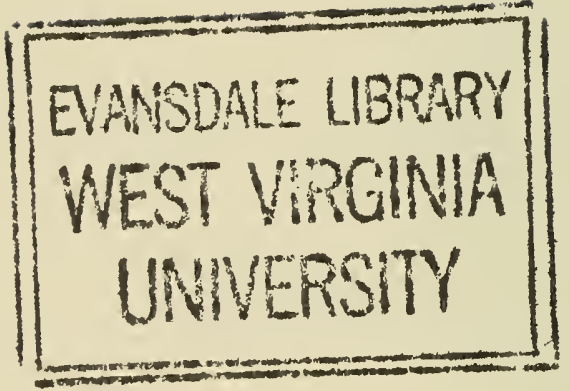




\section{Restricted}

\section{Circulation Only}

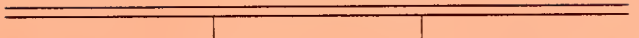




\title{
Aquicultural $\mathbb{E}$ Experintent Station
}

College of Agriculture, rahest Virginia ofnibersity

\author{
HENRY G. KNIGHT, Director \\ Morgantown
}

\section{Some Phases of the Relation of Temperature to the Development of Insects}

(Technical)

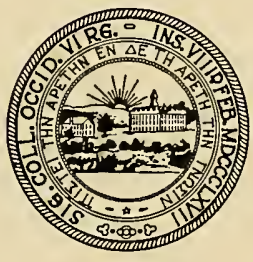

By

L. M. PEAIRS

Publications of this Station will be mailed free to any citizen of West Virginia upon written application. Address Director of the West Virginia Agricultural Experiment Sta. tion, Morgantown, West Virginia. 


\section{AGRICULTURAL EXPERIMENT STATION STAFF}

FRANK BUTLER TROTTER, A. M., LL. D . . . . . . . . . . President of the University HENRY G. KNIGHT, Ph. D............... Dean of the College of Agriculture and Director of the Experiment Station

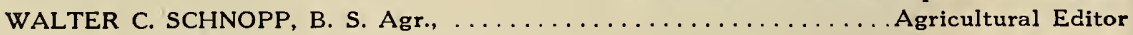

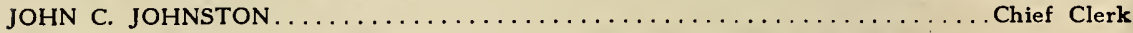

\section{AGRONOMY}

R. J. Garber, Ph. D.

E. P. Deatrick, Ph. D.

Associate Agronomist (Soils)

T. E. Odland, Ph. D.

Associate Agronomist

T. C. Mcllvaine, Ph. D. $\dagger$

Assistant Agronomist (Soils)

D. R. Dodd, M. S.

Assistant Agronomist

M. M. Hoover, M. S.

Junior Agronomist

H. K. Rowley, M. S. Agr.**

Seed Analyst

ANIMAL HUSBANDRY

E. A. Livesay, M. S.

Animal Husbandman

Chas. V. Wilson, M. S.

Assistant Animal Husbandman

J. H. Longwell, M. S.

Assistant Animal Husbandman

J. H. Rietz, D. V. M.

Associate Veterinarian

R. H. Tuckwiller, B. S. Agr.* CHEMISTRY

R. B. Dustman, Ph. D.

Associate Chemist

Chas. E. Weakley, Jr., B. A.

Assistant Chemisı

L. P. Hansen, Ph. D.

Assistant Chemist

T. B. Leith, B. A.** Assistant Chemist

Paul Daughenbaugh

Assistant in Chemistry

\section{DAIRY HUSBANDRY}

E. L. Anthony, M. S. Agr.

Dairy Husbandman

H. O. Henderson, M. S. Agr.

Associate Dairy Husbandman

G. Malcolm Trout, M. S.

Junior Dairy Husbandman

\section{ENTOMOLOGY}

L. M. Peairs, Ph. D.

Entomologist

W. E. Rumsey, B. S.**

L. E. Dills, M. S.**

Assistant in Entomology

\section{FARM ECONOMICS}

A. J. Dadisman, Ph. D.

Farm Economist

Paul A. Eke, Ph. D.

Assistant Farm Economist

F. D. Cornell, Jr., M. S.

Junior Farm Mechanician

W. W. Armentrout, M. S.

Junior Farm Economist

HOME ECONOMICS

Rachel H. Colwell, A. M.

Home Economist

Hazel C. Cameron, M. S.

Research Specialist in Foods

Nell Nesbitt, A. M.

Research Specialist in Home Mgt.

\section{HORTICULTURE}

H. E. Knowlton, Ph. D.

Horticulturist

H. L. Crane, M. S. Agr.

Associate Horticulturist

K. C. Westover, M. S. Agr.

Assistant Horticulturist

Ernest Angelo, M. S. Agr.

Junior Horticulturist

L. F. Sutton, B. S. Agr.

Assistant Horticuiturist

H. P. Sevy, M. S. Agr.

Assistant in Horticulture

M. B. Hoffman, M. S.

Assistant in Horticulture

PLANT PATHOLOGY

N. J. Giddings, Ph. D.

Plant Pathologist

Anthony Berg, M. S.

Assistant Plant Pathologist

L. H. Leonian, Ph. D.

Assistant Plant Pathologist

E. C. Sherwood, M. S.

Assistant Plant Pathologist

\section{POULTRY HUSBANDRY}

Horace Atwood, M. S. Agr.

Poultry Husbandmar

E. T. Wightman, M. S. Agr.

Junior Poultry Husbandmar

RURAL SOCIOLOGY

T. L. Harris, Ph. D.

Rural Sociologis

ZOOLOGY

F. E. Chidester, Ph. D.

Zoologis

* In cooperation with the U. S. Department of Agriculture, Washington, D. C.

tIn charge of the Lakin Sub-Station, Lakin, W. Va.

**In cooperation with the State Department of Agriculture, Charleston, W. Va.

fln charge of the Reymann Memorial Farms, Wardensville, W. Va. 


\section{Some Phases of the Relation of Temperature to the Development of Insects}

Investigators early in the modern scientific era recognized the influence which temperature exerted upon the rate of growth ir plants. Reaumur, as early as 1735 , recognized this relation as a quantitative one and suggested the idea that the total amount of heat, expressed as temperature summations, required to produce a given growth effect, was a constant. The method of summation, as used by Reaumur, assumed that temperatures above the zero of his thermometer, $i$. e., the freezing point of water, were effective while those below zero were not.

Variations in the experimentally determined thermal constants were later associated with the effect of other climatic factors in the processes of growth and led to the search for more accurate methods for the summation of temperatures. Credit appears to be due de Candolle (1832, vide de Candolle 1855) for the statement that effective temperatures do not necessarily persist down to zero but that some point above zero should often be used as the basis for summations of the thermal increment.

From the time of de Candolle's early work progress for some fifty years was mostly in the line of accumulation of statistics in which many investigators participated.

Van Oettingen (1879) seems to have been the first to recognize the straight line relationship of the coefficients of velocity increase in the rate of growth. As the "threshold of development," a term also apparently first used by Van Oettingen, he assumed several possible temperatures, all within the limits of the possible course of the straight line, and from each of these computed the thermal

The project reported in this report was, in its conception, a continuation of work begun by Dr. Dwight Sanderson at the New Hampshire Agricultural Experiment Station, and by him transferred to the West Virginia Agricultural Experiment Station, where it was continued by the writer. During the year 1916-1917 the work was done in the Zoological Laboratories of the University of Chicago. Later it was continued in the entomological laboratory at the West Virginia Agricultural Experiment Station.

Acknowledgements are due to Dr. Sanderson for advice and assistance at the beginning of the project and for continued interest throughout; to the members of the faculty in Zoology at the University of Chicago who have contributed in various ways to the progress of the work. 
increments for each point on the line. The zero point which gave values for the thermal increments at the several points which were most nearly in agreement was assumed to be the actual threshold. This method does not differ in principle from that used in this paper and will give results approximately the same as the method of least squares.

Investigators working with eggs of silk worms early recognized the influence of temperature in the time of hatching (Beauvais, 1837, and numerous others). Whether they or the fish culturists were the first to recognize the influence of temperature on cold blooded animals as similar to that observed with plant growth is not certain but it was early clearly brought out in the works of the fish culturists: Green (1870), Dannevig (1894), Wallich (1900), Reibisch (1902), and others.*

Fere (1894) and Edwards (1902) have shown that warm blooded animals may be subject to a similar effect of temperature variations as they demonstrated that the rate of development of the egg of the domestic fowl depends directly upon the temperature at which it is incubated.

The law of van't Hoff (1884) and Arrhenius' (1889) formula based upon it, demonstrated the relation of the velocity of chemical reaction to the degree of heat and showed that the coefficient for the increase in velocity was frequently between two and three for each ten degree increase in temperature. These formulations, coming as they did at a time when biologists had turned to physics and chemistry for the explanation of vital phenomena, have had a rather profound influence on the interpretations of temperature data from the beginning of the present century. Lillie and Knowlton (1897) showed that the coefficients for the increase in velocity of the development of eggs of Amphibians decreased with increased temperatures and this agrees with the van't Hoff coefficients. Many later workers, mostly in the botanical field, have found that the variations observed in rate of growth can most readily be explained in terms of this law, assuming generally that the coefficient is nearly the same for each ten degree temperature change. Lehenbauer (1914) found no better way to describe his results with the growth of maize seedlings; Livingston applied the law of van't Hoff but, for practical purposes, supplemented it with certain physiological indices furnished by living plants.

${ }^{*}$ Kupfer (1876) denied any influence of temperature upon time of hatching of fish 
Reibisch (1902) in his work with fish eggs described the developmental curve as an hyperbola and calculated, from the formula of the curve, the point which should represent the threshold of development and from which thermal constants or thermal increments should be computed. He showed also that development is possible, with fish egg's of some sorts, below the centigrade zero, provided the density of the solution is great enough to prevent freezing. Some years later Sanderson and Peairs (1914) and Krogh (1914) arrived independently at conclusions essentially identical with those of Reibisch. Krogh and Johannsen (1914), and Krogh (1914) based their conclusions on work with insects, amphibians, echinoderms, and fishes, while Sanderson and Peairs worked with several different kinds of insects.

Loeb and Northrup (1917) showed further evidence of the straight line relationship but their work indicated, as did the earlier work of Krogh, some departure from the straight line condition near the base of the curve. Krafka (1920) discussed the possibility of interpreting this aberration as an entirely different type of curve. It was Shelford (1917) however, who first pointed out the fact that this type of departure from the straight line was rather general and indicated the necessity for a more adequate interpretation of it than had yet been offered.

That temperature affects other vital processes in essentially the same manner as it affects growth and development has been indicated by many investigators. Snyder (1908 and 1911) showed that the conductivity of muscle fibre was affected; Rogers (1911) demonstrated the same for the rate of heart beat; Loeb and Wasteneys (1911) showed that the temperature relation for the oxygen consumption and carbon dioxide production of eggs of Arbacia was similar to the relation of temperature to development; and Krogh (1914 and 1916) showed the same for the carbon dioxide production of insects.

Several other physiological processes have been shown to be affected but the data is not such as to permit the demonstration of the analogy in all cases.

The foregoing statements cannot be said to cover the work which has been done on the subject. Valuable summaries will be found in the works of Bachmetjew (1901 and 1907), Abbe (1905), Kanitz (1915), and Shelford (1917). More or less direct applications of the principles involved will be found in Merriam (1894), 
Hopkins (1900, 1918, and 1919), Sanderson (1908 and 1910), and in many American entomological studies of recent years. Mention of these papers will be found in the bibliography.

\section{EXPERIMENTAL WORK}

\section{Objects}

The experiments herein reported were designed to determine:

1. The developmental curves for insects reared at different constant temperatures.

2. Whether a constant threshold of development can be experimentally demonstrated and, if so, the method for its determination.

3. The validity of the theory of the "Thermal Constant," and assuming such a constant, the correct method for its determination.

4. The differences in the effects of constant temperatures and of variable temperatures upon growth and development.

5. The correlation of the production of carbon dioxide with the rate of development at different temperatures.

6. Whether minor variations in other factors, such as light and moisture, have a sufficiently great influence upon rate of development to affect materially the interpretation of results based upon temperature variations alone.

\section{Explanations of Terms Used}

1. Effective temperature: All temperatures within the experimentally determined range where development may take place.

2. Thermal constant: The total of effective temperatures, expressed in day-degrees, to which the organism is subjected during the developmental period under consideration.

3. Day degrees: One degree of temperature above the zero for the developmental curve, enduring for a period of one day, constitutes a day-degree.

4. Zero of velocity curve: The point at which the curve of velocity of development intersects its temperature axis.*

This is the "Developmental Zern" of Sanderson and Peairs (1914) and has been termed "Physiological zero", "Critical point", and "Threshold of development." All of these terms, used in this connection, are inexact and capable of misinterpretation. 
5. Threshold of development: This term indicates the temperature at which, on the descending scale, development definitely ceases and at which, on the ascending scale, development is again initiated. This point may or may not correspond with the zero of the velocity curve but since there is evidence which indicates that the two points are not the same in all cases, it is desirable to keep the terms separate. The term is here used in the sense of Shelford, Reibisch, and Von Oettingen.

6. Hyperbola: A curve in which the product of the two factors upon which any point upon the curve is based is constant. The formula for such a curve is $\mathrm{X} \times \mathrm{Y}=\mathrm{C}$.

7. Reciprocal curve: These are formed by plotting the reciprocal value of the time factor against the temperature factor. Such a reciprocal value of the time factor expresses the value of one day in terms of percent of development. The reciprocal for a perfect hyperbola is a straight line. The approach of the points on the reciprocal curve to the straight line, therefore, indicates the relation of the developmental curve to the true hyperbola. This curve shows the rate of acceleration of development.

8. Index line, or index of development: This is the same as the reciprocal curve. Any point on this line indicates the rapidity of development at the temperature corresponding.

9. Factor or index number: The value of one day expressed in percent of the whole period required. This may be read directly on the index line.

10. Exponential curve: A curve whose points are established by treating the time values, determined experimentally, with some factor or exponent so that their relative magnitude is changed. Such a curve would be necessary to express the velocity of development if the value of a degree at one point within the developmental range should prove to be different from that of a degree at some other point. In such event, factorial treatment of the recorded temperatures would be necessary in order to derive the true constant.

11. Optimum temperature: The temperature at which the greatest percentage of insects are able to complete normal development. This is not usually the temperature at which development takes place with the greatest velocity. 


\section{Methods of Presenting the Data}

The most convenient and useful methods of presenting data of the type included in this report is by means of tables and graphs. Explanations accompany the tabulated material and the method of constructing the graphs follows.

Curves showing the relation of the time factor to the temperature at which development takes place were constructed directly from the data and have been found to approximate the hyperbola in form. A more instructive presentation, and the one here followed, has been to plot the reciprocals of the time factor, showing the comparative values of a day at the different temperatures. Such curves show directly the relation between the increase in velocity of development and change in temperature. Inspection of these curves shows that they approach the straight line; it has seemed. more satisfactory to show the relation of the points of the curves to a straight line than to plot the actual curves. The best fitting straight line has, therefore, been determined by the method of "least squares." Such straight lines have been considered, in discussions of the data, as the curves of velocity of development. The points on all curves have been established from points which represent weighted averages. These give due consideration to the varying numbers of individuals in each emergence record. Any point, as finally established is a component of all emergence records for the temperature. Similarly, each point is given a weighted value in the determination of the course of the final best-fitting straight line.

The zero point, as has been stated before, used in the calculation of thermal increments, is that point at which the straight line curve thus established, intersects the temperature axis.

\section{Description of Experiments}

\section{MATERIALS USED}

Experimental work involved in this paper deals with insects of the following groups:

Lepidoptera: the codling moth, Carpocapsa pomonella Linn., pupae; the corn earworm, Chloridea obsoleta Fabr., larvae and pupae; the cabbage butterfly, Pontia rapae L., Iarvae and pupae; Malacosoma americana Fabr., the tent caterpillar, pupae; Coleoptera: the Colorado potato beetle, Leptinotarsa 10-lineata Say, egg: larvae, and pupae; Diptera: the house fly, Musca domestica L., larvae and pupae; Lucilia caesar L., the green-bottle fly, larvae and pupae; 
Calliphora vomitoria L., the bluebottle, larvae and pupae; Sarcophaga carnaria L., (?) a flesh fly, larvae and pupae.

In addition to these, many insects were studied which do not appear in this report for the reason, usually, that not enough points on the developmental curve were established to make the results of value in the conclusions drawn. None were omitted because preliminary evidence was of a discordant nature.

\section{SOURCES OF EXPERIMENTAL MATERIAL}

All insects used in the experiments reported herein have been collected at Morgantown, West Virginia. Fly eggs were collected from meat exposed for that purpose. The collections of this material extended over a period of several years and may be supposed to represent all the usual variations to be encountered in field collected material which may be due to seasonal variation, variation from year to year, and individual variation in the parent insects. Larvae were hatched in the laboratory from the eggs thus collected. The time of hatching of all eggs used in experimental work was recorded accurately within one hour. Pupae were usually secured from cultures allowed to develop on food provided but kept under outdoor conditions; only rarely were pupae from incubator raised material used. When such pupae were used they were always from the incubators within which moderate temperatures were maintained, e.g., $15^{\circ}$ or $20^{\circ}$. The time of pupation was known always within three hours, usually within one or two hours, and always within one hour when the insects were to be placed in incubators at high temperatures.

Sarcophaga larvae were taken from exposed meat where the time of their deposition was known within one hour.

Chloridea obsoleta larvae were collected in the field and the age of the young larvae was not definitely known but all those used were less than one-fourth inch in length and as nearly uniform in size as it was possible to secure them. Pupae of this insect were from larvae collected in the field but allowed to pupate in the laboratory.

Carpocapsa pomonella pupae were from larvae collected during June and July and were all, presumably, those which form what is known as the first brood. Time of pupation was observed within three hours in all material used.

Malacosoma americana pupae used were from larvae reared in the laboratory, the time of pupation of which was known within six hours. 
Leptinotarsa 10-lineata eggs used were those the deposition of which had been observed and larvae were from such eggs hatched in the laboratory. Pupae were from field collected larvae allowed to pupate in the laboratory.

\section{APPARATUS USED}

Specially constructed incubators of square form and having a capacity of about two cubic feet were used throughout the work. Those designed to maintain temperatures above room temperature were kept in the laboratory; those for temperatures of $15^{\circ}$ and $20^{\circ}$ were kept inside a large refrigerator while those at $12^{\circ}$ and lower temperatures were kept in a cold storage room during the greater part of the course of the experiments although at times these also were kept in the large refrigerator.

Heat was supplied by small electric light bulbs, blackened. Two and four candle power, carbon filament bulbs were used. In order to secure uniform distribution of the heat four or six of these bulbs were used in each incubator. The bulbs were so placed as to give the most even distribution of the heat throughout the chamber. Heat control was by means of thermostats, several types having been used but none giving better control than a small, simple, bi-metallic type. These maintained temperatures which did not vary, in any of the experiments recorded, more than about one-half degree centigrade above or below the desired temperature.

Thermometers of several types were used. Each incubator was supplied with a maximum and minimum thermometer calibrated against a Bureau of Standards certified thermometer. These indicated merely the variation, the temperature being recorded from a large thermometer reading to tenths of degrees and kept with its bulb as nearly as possible in the center of the insect containers in the incubator at the time. At intervals of about a week the variability of the temperature was further checked by keeping a thermograph in the incubator for two days.

No records were kept of material when the temperature varied more than one degree Fahrenheit above or below the desired temperature so the experimental error due to temperature variations may be considered as less than five-ninths of one degree Centigrade. The method of recording the temperatures from the part of the incubator in which the insect material was located reduces the possibility of error from variations within the incubator although repeated tests of the temperature in different parts of the incubators showed that such variation was never great. 
Material within the incubators was always so supported as to allow free circulation of air below and around it but no other provision was usually made to secure circulation. In early tests circulation was provided by small electric fans but since no apparent difference in results was obtained by this method it was discontinued.

No attempt was made to keep the relative humidity constant but it was usually maintained at near the point of saturation. Wet and dry bulb thermometers were used to measure the degree of moisture. Semi-automatic devices feeding water onto large wicks suspended around the heating bulbs supplied atmospheric moisture. In some of the experiments with caterpillars it was found that the moisture was injurious and the incubators were allowed to become somewhat dryer inside, the moisture ranging from not less than 40 to about 65 per cent relative humidity.

In practically all the work reported the insects were kept in complete darkness or in very dim light.

\section{METHODS OF HANDLING MATERIAL}

Since the bulk of the material used in these studies was fly larvae and pupae, the description of methods here given applies to that material. In case methods were varied in the manipulation of the material, mention is made of the variation.

Fly larvae, excepting Sarcophaga, were secured from eggs hatched in the laboratory, as has been stated. Usually the hatching was actually observed so that the time was known exactly; in a few instances the time was not known within from one to three hours. Ordinarily such material was placed in the cold incubators so that the slight variable introduced would have proportionately less effect in the result since the developmental period in the cold incubators was so long that the one or two hours variation at the start would be "absorbed" without affecting materially the value of the results. No larvae were permitted to feed before being placed in the incubators. This further reduced the chance for any effect from variation in the length of time from hatching to entrance into the incubators.

Food supplied for the fly larvae was lean beef of which an excess was always furnished. When the feeding period occupied less than one week this was not renewed. Usually when the period of feeding extended for more than a week the food was changed 
once in about five days. Larvae eating foliage were supplied with fresh food once or more each day and were transferred by hand from the old food to the fresh.

Food for the flies was half buried in moist sand and kept covered with moist blotting paper. Various containers were tried but the ones most used were tubes one inch by three inches with bottoms of plaster of paris about one-fourth inch thick. These were sunk in moist sand, the plaster bottoms of the tubes usually absorbing moisture enough to keep the sand within them in moist condition. The tubes were stoppered with cotton or with perforated plaster. From twenty-five to one hundred larvae, usually the smaller number, were kept in each tube. When all had ceased to feed and entered the "pre-pupal stage," the food was removed and sand added. Pupation took place in the sand and observations were so arranged that the time of pupation was recorded within six hours, except in lots where the development extended over periods exceeding twenty days when the observations were sometimes made only twice daily.

Pupae were kept in tubes similar to those in which the larvae were reared or in broad flat cylinders with plaster bottoms and wire screen covers. About one inch depth of sand was kept in the bottoms of the large containers and somewhat more in the smaller ones. The pupae were covered with about one-half inch of moist sand. Emergence records were kept for the pupae similar to the pupation record for the larvae.

All larvae and pupae were counted although it was found that sufficiently accurate estimations of the numbers might have been secured by weighing.

The sand in the breeding containers was generally kept sufficiently moist by absorption through the plaster bottoms from the wet sand outside but daily examination was made and when they were apparently not absorbing enough water more was added directly, so that the medium, food or sand, was moist at all times. Since the sand used was always sterilized by dry heat and only distilled water was used little trouble from molds was encountered although this treatment did not provide entirely aseptic conditions.

Larger containers, usually lantern globes, were used for caterpillars and their pupae.

Codling moth larvae were placed between sheets of glass separated by about the thickness of the larvae so that their pupation 
could be observed directly without removing them from their cocoons for examination.

Throughout the work, when temperature or other conditions within the incubator were not according to the standard, or when observations were missed during a critical period so that there was any doubt as to the duration of the stage, the time of first emergence, or any other important fact, the lots of insects involved were discarded.

Numbers of insects used are indicated in the tabulated summaries of results. These numbers were large enough in most cases, so that minor variations in material or occasional errors of observations should not affect the general results.

Conditions approximating variable temperature conditions were secured by moving insects from one constant temperature chamber to another at intervals of six or twelve hours. Less frequent changes did not, apparently, furnish conditions comparable to outdoor condition.*

Outdoor conditions were maintained in a screened breeding cage kept in a shaded situation and having the temperature recorded by a thermograph.

\section{EXPERIMENTAL DATA}

\section{Constant Temperatures}

In the experiments with constant temperatures, insects were maintained at different constant temperatures, each lot being kept at one temperature throughout its period of development. Temperatures ranging from $5^{\circ} \mathrm{C}$. to $35^{\circ} \mathrm{C}$. at five degree intervals, with a few lots at $6.5^{\circ}, 8^{\circ}, 12^{\circ}$, and $40^{\circ}$ are reported. Many low temperature lots from temperatures which later were found to be below the threshold of development have been omitted from the summaries because they showed no evidence of development. The same is true for high temperatures. Evidence of considerable development at $40^{\circ}$ or higher temperatures was rare and no mention is made of the many lots incubated at such points.

Data are available usually for the establishment of six or more points upon a developmental curve. A considerable number of intermediate points have been established by observations of insects in much smaller numbers than are represented by most of the lots - reported. These have been omitted because the numbers are not

*A special thermostat so constructed as to provide temperatures varying in any lesired manner during the day was devised but some difficulties in cooling the incubator were encountered; later these were overcome but no insects reared in this incubator are
zere recorded. 
comparable to the others and because they do not add anything of importance to the report.

The results reported, based upon work with the four species of flies, may be considered as the most significant part of the evidence because of the number of insects involved and because the possibility of variation in factors other than temperature was less with the fly material.

The results are presented in tabular form on the following pages. The presentation, in connection with the tables, of "Thermal Constants" and of temperature, or effective temperature, summations based upon the interpretation of the velocity curve as a straight line should not be taken to imply a fixed preformed conviction that this was the correct interpretation. It has merely been found convenient, in the tables and in the graphs, to show the relation of the experimentally established points to the straight line and the relation of the termal increments to a constant. What the significance of these relations may be will be brought out in the discussion of the data.

The possibility that the time of first emergence of individuals at different temperatures may be a more exact index of the effect of temperature than the mean emergence time has led to the inclusion of summaries based on first emergence along with those based upon mean emergence. This first emergence data represents the average time of first emergence, usually from many separate lots of individuals subjected to the same treatment, rather than the absolute minimum emergence time.

In the tabular summaries are given the temperatures; the developmental time in days; the accumulated temperatures based upon the summation of daily temperatures above the zero point indicated by the velocity curve, accumulated temperatures and thermal constants being expressed throughout this work in daydegrees; the standard deviation (also in day-degrees) for the developmental time factor based upon all individuals emerging; the standard deviation for the accumulated temperature based upon the averages for the several separate lots comprised in each summary; standard deviations for time and for the thermal increment based upon first emergences calculated in the same manner as those for the mean emergences; and the index of development based on mean emergence as well as that based upon the first emergence. Thermal constants based upon the mean emergence velocity curve and upon the first emergence velocity curve are also given. 
TABLE 1.-Lucilia caesar, larvae. Constant temperatures (See Fig. 1).

\begin{tabular}{|c|c|c|c|c|c|c|c|c|c|c|c|}
\hline \multirow{2}{*}{ T. } & \multirow{2}{*}{$\begin{array}{l}\text { No. of } \\
\text { Insects }\end{array}$} & \multirow{2}{*}{$\begin{array}{l}\text { Perc't. } \\
\text { Devel- } \\
\text { oped }\end{array}$} & \multicolumn{3}{|c|}{$\begin{array}{c}\text { Length of Stage in } \\
\text { Days }\end{array}$} & \multicolumn{4}{|c|}{ Acc. Effect. Temp. } & \multicolumn{2}{|c|}{ Index of Dev. } \\
\hline & & & Ave. Min. & Mean & S. D. & Mean & S. D. & Min. & S. D. & Mean & Min. \\
\hline $35^{\circ}$ & 14,654 & 46 & 3.5 & 3.7 & .21 & $109^{\circ}$ & $63^{\circ}$ & $102.5^{\circ}$ & $3.1^{\circ}$ & 270 & 28.6 \\
\hline 30 & 15,100 & 71 & 4.2 & 4.4 & .23 & 108 & 56 & 102.1 & 2.2 & 22.5 & 23.8 \\
\hline 25 & 14,120 & 79 & 5.25 & 5.3 & .27 & 103 & 52 & 103.2 & 2.0 & 186 & 190 \\
\hline 20 & 17,340 & 89 & 7.0 & 7.4 & .18 & 107 & 26 & 100.1 & 3.1 & 136 & 14.3 \\
\hline 15 & 11,600 & 84 & 11.1 & 12.0 & .29 & 114 & 4.7 & 1032 & 1.9 & 8.4 & 90 \\
\hline 10 & 12,300 & 54 & 24.9 & 26.5 & 1.2 & 119. & 5.4 & 1071 & 3.3 & 3.9 & 40 \\
\hline 8 & 4,300 & 36 & 47.4 & 49.5 & 3.0 & 123 & 7.6 & 109.0 & 4.2 & 20 & 2.1 \\
\hline 6.5 & 2,000 & 5.2 & 135. & 136. & 2.2 & 136 & 2.2 & 108.0 & 1.9 & .73 & .74 \\
\hline 5 & 5,400 & 0 & & & & & & & & & \\
\hline
\end{tabular}

Thermal constant of curve: Mean, $110.7^{\circ}$, S. D., $3.9^{\circ}$; Ave. Min., $103.0^{\circ}$, S. D., 2.6 $6^{\circ}$ Zero of curve: Mean, $5.5^{\circ}$; Ave. Min., $5.7^{\circ}$.

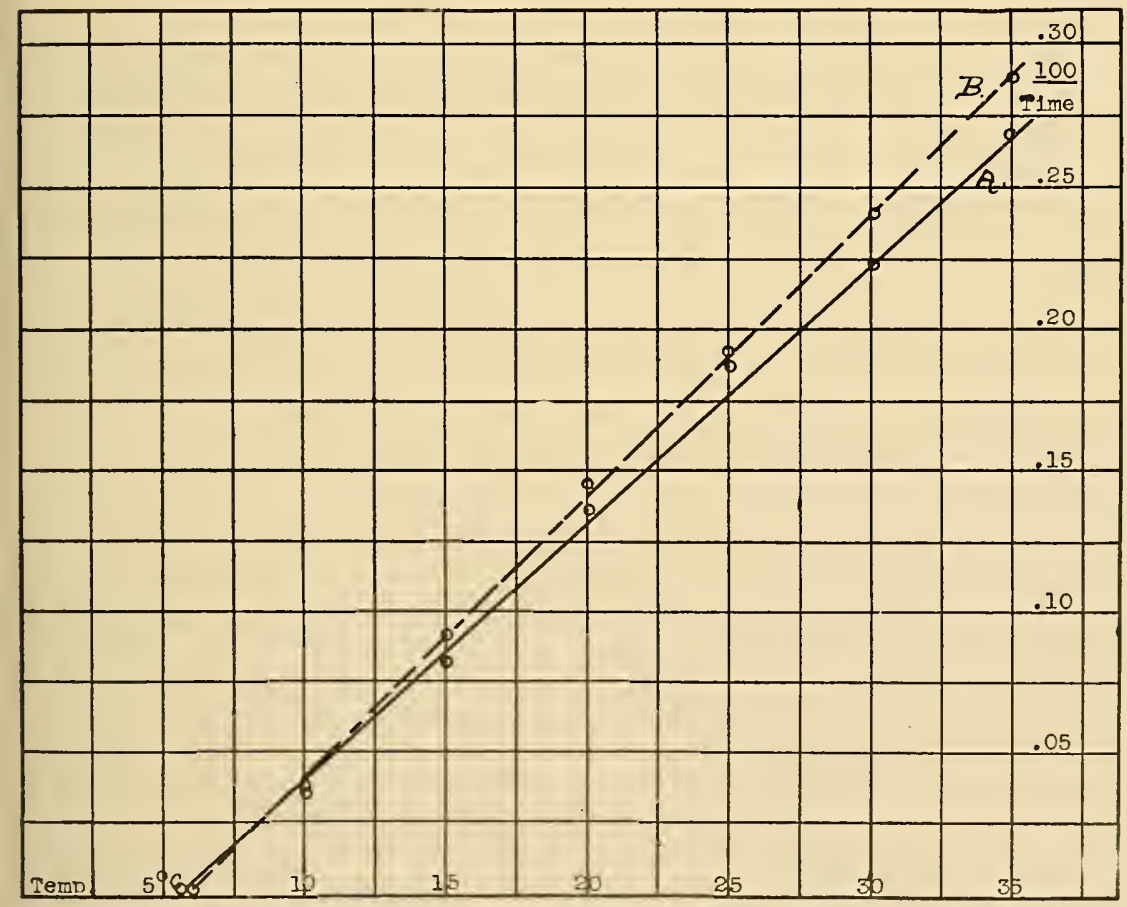

Fig. 1.-Velocity of development of Lucilia caesar larvae. A.-Based upon the mean time of emergence. B.-Based upon the average time of first emergence for the different lots. (See Table 1.) 
TABLE 2.-Lucilia caesar, pupae. Constant temperatures (See Fig. 2).

\begin{tabular}{|c|c|c|c|c|c|c|c|c|c|c|c|}
\hline \multirow{2}{*}{$\mathbf{T}$. } & \multirow{2}{*}{$\begin{array}{l}\text { No. of } \\
\text { Insects }\end{array}$} & \multirow{2}{*}{$\begin{array}{c}\text { Perc't. } \\
\text { Devel- } \\
\text { oped }\end{array}$} & \multicolumn{3}{|c|}{$\begin{array}{c}\text { Length of Stage in } \\
\text { Days }\end{array}$} & \multicolumn{4}{|c|}{ Acc. Effect. Temp. } & \multicolumn{2}{|c|}{ Index of Dev. } \\
\hline & & & Ave. Min. & Mean & S. D. & Mean & S. D. & Min. & S. D. & Mean & Min. \\
\hline $35^{\circ}$ & 12,400 & 57 & 3.9 & 4.2 & .31 & $121^{\circ}$ & $8.8^{\circ}$ & $110.0^{\circ}$ & $3.6^{\circ}$ & 24.0 & 25.6 \\
\hline 30 & 14,200 & 65 & 4.6 & 5.1 & .34 & 119 & 8.0 & 106.0 & 2.2 & 19.5 & 21.8 \\
\hline 25 & 9,800 & 81 & 5.7 & 6.5 & .46 & 120 & 8.5 & 103.7 & 2.0 & 15.5 & 17.5 \\
\hline 20 & 13,900 & 89 & 7.8 & 9.2 & .86 & 125 & 11.6 & 103.0 & 2.3 & 10.8 & 12.8 \\
\hline 15 & 9,200 & 83 & 12.3 & 14.5 & 1.63 & 123 & 13.8 & 100.9 & 3.0 & 6.8 & 8.1 \\
\hline 10 & 8,000 & 67 & 34.0 & 38.0 & 3.34 & 133 & 12.0 & 108.8 & 4.4 & 2.6 & 2.9 \\
\hline 8 & 1,600 & 7 & 94.8 & 100.0 & 14.70 & 150 & 22.0 & 113.8 & 5.2 & 1.0 & 1.1 \\
\hline 5 & 4,000 & 0 & & & & & & & & & \\
\hline
\end{tabular}

Thermal constant of curve: Mean, $122.8^{\circ}$, S. D., $9.2^{\circ}$; Ave. Min., $104.8^{\circ}$, S. D., $3.6^{\circ}$. Zero of curve: Mean, $6.5^{\circ}$; Ave. Min., $6.8^{\circ}$.

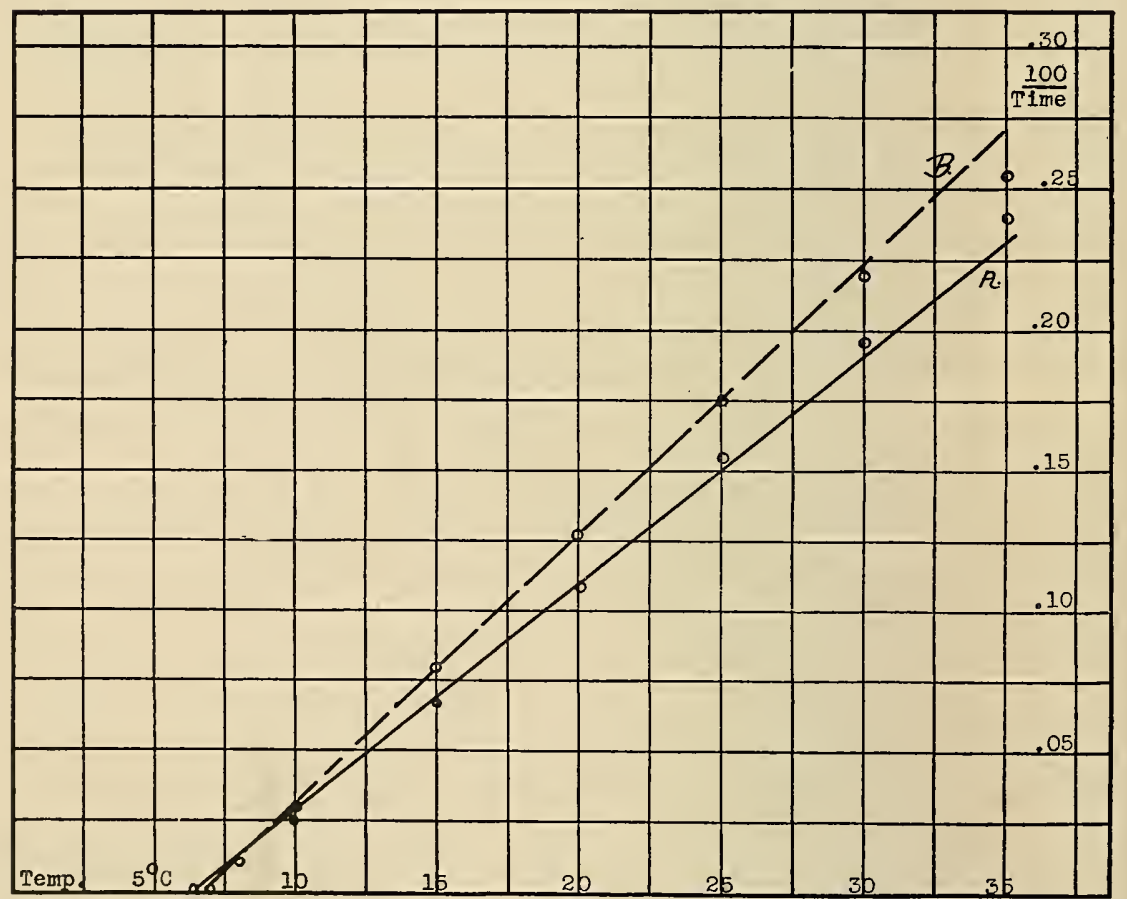

Fig. 2.-Velocity of development of Lucilia caesar pupae. A.-Based upon the time of emergence. B.-Based upon the average time of first emergence for the different lots. (See Table 2.) 
TABLE 3.-Calliphora vomitoria, larvae. Constant temperatures (See Fig. 3).

\begin{tabular}{|c|c|c|c|c|c|c|c|c|c|c|c|c|}
\hline \multirow{2}{*}{$\mathrm{T}$. } & \multirow{2}{*}{$\begin{array}{l}\text { No. of } \\
\text { Insects }\end{array}$} & \multirow{2}{*}{$\begin{array}{c}\text { Perc't. } \\
\text { Devel- } \\
\text { oped }\end{array}$} & \multicolumn{4}{|c|}{$\begin{array}{c}\text { Length of Stage in } \\
\text { Days }\end{array}$} & \multicolumn{4}{|c|}{ Acc. Effect. Temp. } & \multicolumn{2}{|c|}{ Index of Dev. } \\
\hline & & & Ave. Min. & Mean & S. D & & Mean & S. D. & Min. & S. D. & Mean & Min. \\
\hline $35^{\circ}$ & 9,800 & 39 & 3.2 & 3.4 & & 46 & $98.6^{\circ}$ & $4.3^{\circ}$ & $93.4^{\circ}$ & $2.2^{\circ}$ & 29.0 & 31.2 \\
\hline 30 & 8,400 & 64 & 3.9 & 4. 4 & & 31 & 105.6 & 5.2 & 94.4 & 2.0 & 23.0 & 25.6 \\
\hline 25 & 14,000 & 83 & 4.8 & 5.4 & & 29 & 104.5 & 4.7 & 92.16 & 2.6 & 18.5 & 20.8 \\
\hline 20 & 14,700 & 92 & 6.6 & 7.3 & & 33 & 102.2 & 3.9 & 93.7 & 18 & 13.7 & 15.1 \\
\hline 15 & 11,400 & 82 & 10.5 & 11.7 & & 98 & 105.0 & 4.9 & 96.6 & 3.3 & 8.1 & 9.5 \\
\hline 10 & 10,900 & 59 & 23.0 & 27.3 & 2. & 00 & 109.2 & 4.6 & 96.6 & 4.1 & 3.7 & 43 \\
\hline 8 & 2,350 & 33 & 44.6 & 56.0 & 5. & 80 & 112.0 & 6.1 & 98.1 & 3.8 & 1.8 & 2.2 \\
\hline 5 & 3,300 & 0 & & & & & & & & & & \\
\hline
\end{tabular}

Thermal constant of curve: Mean, $104.7^{\circ}$, S. D., $6.9^{\circ}$; Ave. Min., 94.5०, S. D., 3.1 $1^{\circ}$ Zero of curve: Mean, $6^{\circ}$; Ave. Min., $5.8^{\circ}$.

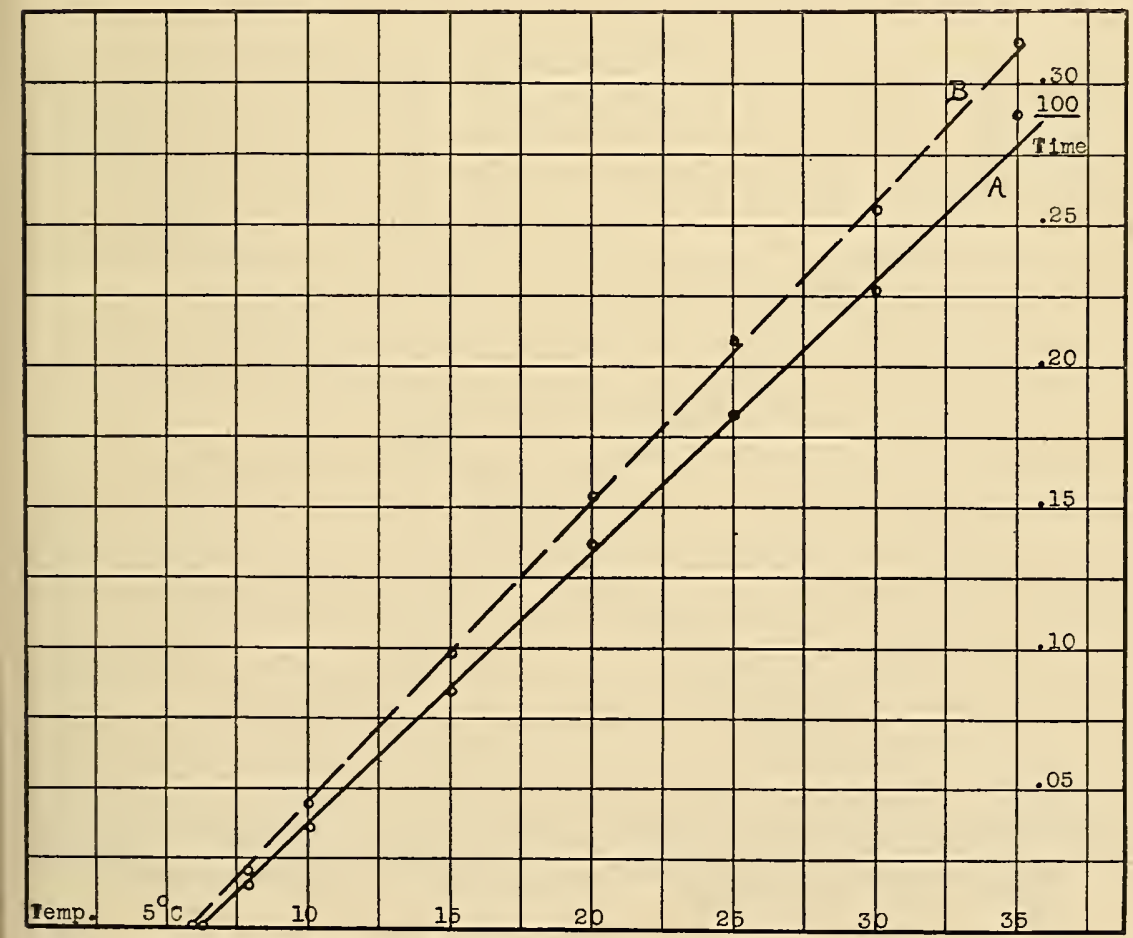

Fig. 3.-Velocity of development of Calliphora vomitoria larvae. A.-Based upon the mean time of emergence. B.-Based upon the average time of first emergence for the different lots. (See Table 3.) 
TABLE 4.-Calliphora vomitoria, pupae. Constant temperatures (See Fig. 4).

\begin{tabular}{|c|c|c|c|c|c|c|c|c|c|c|c|}
\hline \multirow{2}{*}{ T. } & \multirow{2}{*}{$\begin{array}{l}\text { No. of } \\
\text { Insects }\end{array}$} & \multirow{2}{*}{$\begin{array}{c}\text { Perc't. } \\
\text { Devel- } \\
\text { oped }\end{array}$} & \multicolumn{3}{|c|}{$\begin{array}{c}\text { Length of Stage in } \\
\text { Days }\end{array}$} & \multicolumn{4}{|c|}{ Acc. Effect. Temp. } & \multicolumn{2}{|c|}{ Index of Dev. } \\
\hline & & & Ave Nin. & Mean & S. D. & Mean & Min. & S. D. & S. D. & Mean & Min. \\
\hline $35=$ & $7.000^{\circ}$ & 54 & 42 & 4.6 & 1.9 & $126.0^{\circ}$ & 4. $7^{\circ}$ & $116.3^{\circ}$ & $3.2^{\circ}$ & 21.8 & 23.8 \\
\hline 30 & 9,500 & 63 & 5.1 & & .33 & 133.4 & 5.5 & 115.8 & 4.2 & 17.2 & 19.6 \\
\hline 25 & 9,950 & 82 & 6.5 & 70 & 31 & 126.0 & 2.5 & 114.0 & 3.0 & 14.3 & 15.3 \\
\hline 20 & 11,650 & 92 & 8.9 & 9.4 & .70 & 124.0 & 7.1 & 113.0 & 3.3 & 10.6 & 11.2 \\
\hline 15 & 10,100 & 87 & $1 j$ & 16.9 & 1. 49 & 135.2 & 8.3 & 115.5 & 4.2 & 5.9 & 6.7 \\
\hline 10 & 10,500 & 43 & 44.3 & 46.7 & 45 & 140.1 & 12.6 & 119.6 & 7.1 & 2.1 & 2.2 \\
\hline 6 & 3,000 & 1 & 156.2 & 157 & .19 & 157.3 & 4 & 109.3 & .1 & .6 & .63 \\
\hline 5 & 4,300 & 0 & & & & & & & & & \\
\hline
\end{tabular}

Thermal constant of curve: Mean, $130.1^{\circ}$, S. D., 5.7 ; Ave. Min., 114.8, S. D., 2.3 $3^{\circ}$. Zero of curve: Mean, $7^{\circ}$; Ave. Min., $7.3^{\circ}$.

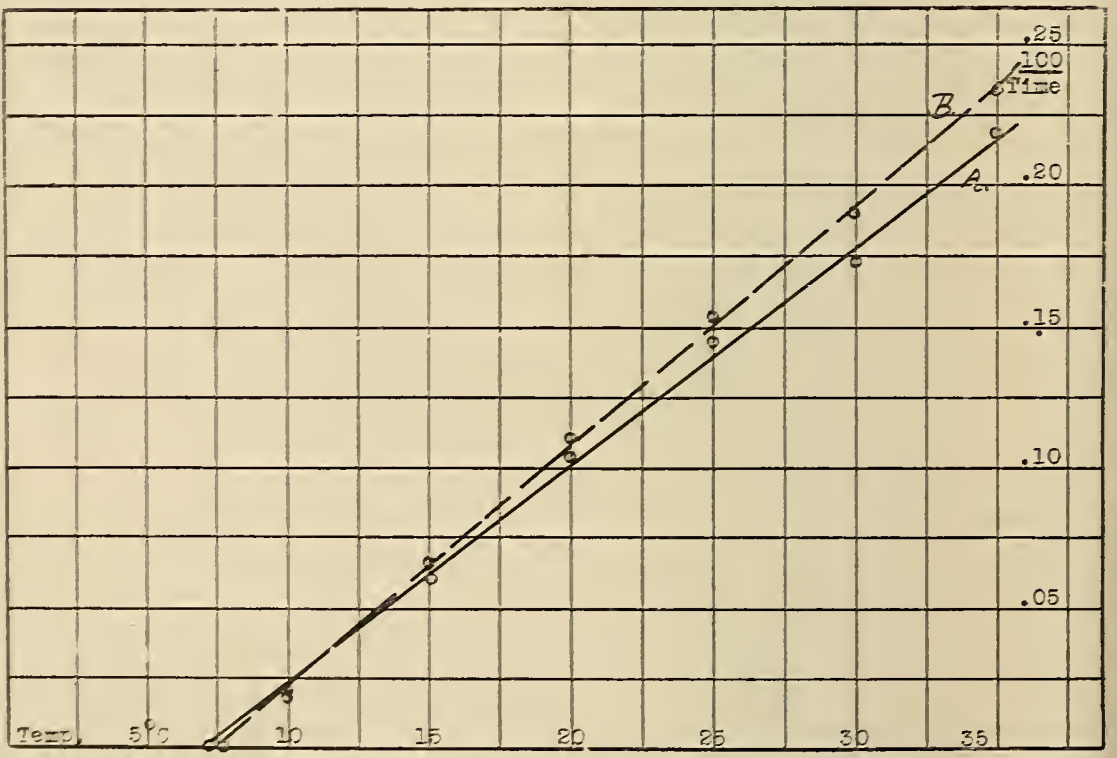

Fig. 4.-Velocity of development of Calliphora vomitoria pupea. A.-Based upon the mean time of emergence. B.-Based upon the average time of first emergence for the different lots. (See Table 4.) 
TABLE 5.-Musca domestica, larvae. Constant temperatures (See Fig. 5).

\begin{tabular}{|c|c|c|c|c|c|c|c|c|c|c|c|}
\hline \multirow{2}{*}{ T. } & \multirow{2}{*}{$\begin{array}{l}\text { No. of } \\
\text { Insects }\end{array}$} & \multirow{2}{*}{$\begin{array}{c}\text { Perc't. } \\
\text { Devel- } \\
\text { oped }\end{array}$} & \multicolumn{3}{|c|}{$\begin{array}{c}\text { Length of Stage in } \\
\text { Days }\end{array}$} & \multicolumn{4}{|c|}{ Acc. Effect. Temp. } & \multicolumn{2}{|c|}{ Index of Dev. } \\
\hline & & & Ave Min. & Mean & S. D. & Mean & S. D. & Min. & S. D. & Mean & Min. \\
\hline $35^{\circ}$ & $2,5 j 0$ & 75 & 4.4 & 45 & 27 & $128^{\circ}$ & $54^{\circ}$ & $12 j 4^{\circ}$ & $23^{\circ}$ & 220 & 227 \\
\hline 30 & 2,000 & 86 & 5.3 & 5.6 & 36 & 133 & 54 & $12+6$ & 21 & 180 & 18.9 \\
\hline 25 & 2,024 & 94 & 6.8 & 72 & 41 & 133 & +9 & 125.8 & 30 & 139 & $1+7$ \\
\hline 20 & 2,500 & 97 & 9.0 & 9.6 & 39 & 130 & 5.3 & 121.5 & 24 & 104 & 11.1 \\
\hline 15 & 2,412 & 78 & 14.4 & 15.2 & 92 & 129 & 69 & 122.4 & 36 & 6.7 & 6.9 \\
\hline 10 & 2,016 & 72 & 33.1 & 34.0 & 4.7 & 119 & 16.7 & 115.65 & +2 & 29 & 30 \\
\hline 8 & 500 & 6 & 90.1 & 903 & .9 & 136 & 1.6 & 133.1 & .1 & 1.1 & 1. 1 \\
\hline 5 & 2,300 & 0 & & & & & & & & & \\
\hline
\end{tabular}

Thermal constant of curve: Mean, $129.9^{\circ}$, S. D., $6.9^{\circ}$; Ave. Min., $124.3^{\circ}$, S. D., 3.8 ${ }^{\circ}$ Zero of curve: Mean, $6.5^{\circ}$; Ave. Min., $6.5^{\circ}$.

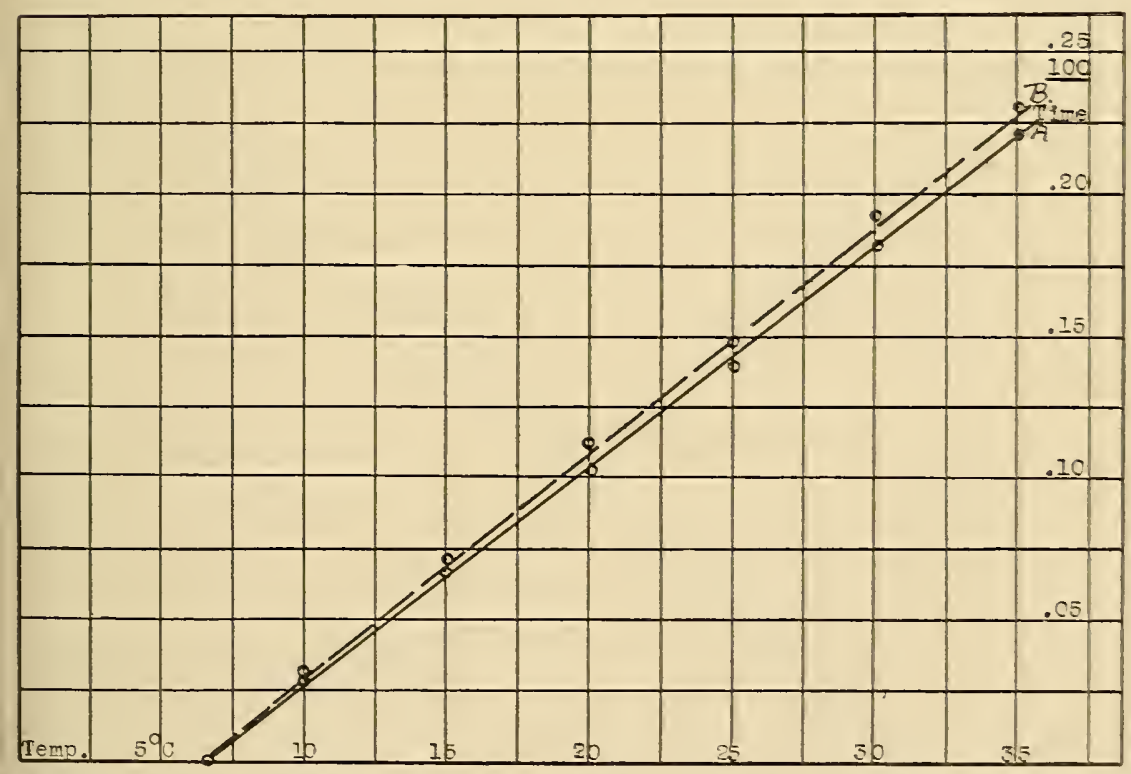

Fig. 5.-Velocity of development of Musca domestica larvae. A.-Based upon the mean time of development. B.-Based upon the average time of first emergence for the different lots. (See Table 5.) 
TABLE 6-Musca domestica, pupae. Constant temperatures (See Fig. 6).

\begin{tabular}{|c|c|c|c|c|c|c|c|c|c|c|c|}
\hline \multirow{2}{*}{ T. } & \multirow{2}{*}{$\begin{array}{l}\text { No. of } \\
\text { Insects }\end{array}$} & \multirow{2}{*}{$\begin{array}{c}\text { Perc't. } \\
\text { Devel- } \\
\text { oped }\end{array}$} & \multicolumn{3}{|c|}{$\begin{array}{l}\text { Length of Stage in } \\
\text { Days }\end{array}$} & \multicolumn{4}{|c|}{ Acc. Effect. Temp. } & \multicolumn{2}{|c|}{ Index of Dev. } \\
\hline & & & Ave. Min. & Mean & S. D. & Mean & S. D. & Min. & S. D. & Mean & Min. \\
\hline $35^{\circ}$ & 1,470 & 65 & 3.7 & 4.0 & .15 & $112^{\circ}$ & $2.1^{\circ}$ & $107.2^{\circ}$ & $1.1^{\circ}$ & 25.0 & 26.3 \\
\hline 30 & 2,900 & 88 & 4.6 & 4.9 & .26 & 113 & 1.9 & 106.7 & .4 & 20.4 & 21.7 \\
\hline 25 & 2,644 & 87 & 5.6 & 5.9 & .44 & 106 & 3.6 & 101.9 & 2.9 & 17.0 & 17.9 \\
\hline 20 & 3,025 & 96 & 7.8 & 8.25 & .80 & 107 & 7.4 & 103.0 & 3.6 & 12.1 & 12.8 \\
\hline 15 & 2,650 & 83 & 13.2 & 15.4 & 1.23 & 123 & 13.0 & 108.2 & 5.0 & 6.5 & 7.6 \\
\hline 10 & 3,000 & 71 & 32.2 & 35.7 & 4.2 & 107 & 12.5 & 103.0 & 3.1 & 2.9 & 3.1 \\
\hline 8 & 600 & 20 & 92.0 & 127.0 & 11.1 & 127 & 11.1 & 110.4 & $\ldots$ & .8 & 1.08 \\
\hline 5 & 1,140 & 0 & & & & & & & & & \\
\hline
\end{tabular}

Thermal constant of curve: Mean, $112.7^{\circ}$, S. D., 7.1 ${ }^{\circ}$; Ave. Min., 105.3 S. D., $3.3^{\circ}$. Zero of curve: Mean, $7^{\circ}$; Ave. Min., $6.8^{\circ}$.

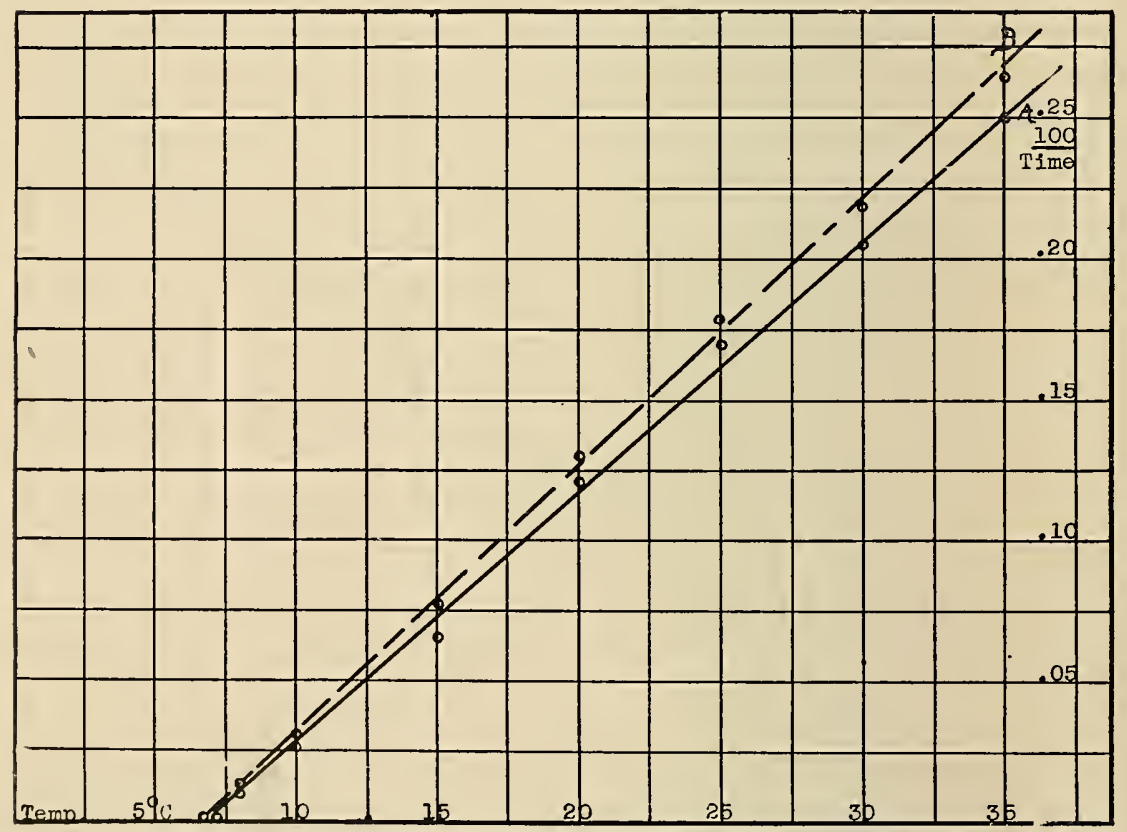

Fig. 6.-Velocity of development of Musca domestica pupae. A.-Based upon the mean time of emergence. B.-Based upon the average time of first emergence for the different lots. (See Table 6.) 
TABLE 8.-Sarcophaga carnaria (?), pupae. Constant temperatures (See Fig. 8).

\begin{tabular}{|c|c|c|c|c|c|c|c|c|c|c|c|}
\hline \multirow{2}{*}{ T. } & \multirow{2}{*}{$\begin{array}{l}\text { No. of } \\
\text { Insects }\end{array}$} & \multirow{2}{*}{$\begin{array}{c}\text { Perc't. } \\
\text { Devel- } \\
\text { oped }\end{array}$} & \multicolumn{3}{|c|}{$\begin{array}{c}\text { Length of Stage in } \\
\text { Days }\end{array}$} & \multicolumn{4}{|c|}{ Acc. Effect. Temp. } & \multicolumn{2}{|c|}{ Index of Dev. } \\
\hline & & & Ave. Min. & Mean & S. D. & Mean & S. D. & Min. & S. D. & Mean & Min. \\
\hline $40^{\circ}$ & 300 & 29 & 3.75 & 3.8 & .12 & $127^{\circ}$ & $3.9^{\circ}$ & $113.6^{\circ}$ & $1.7^{\circ}$ & 25.4 & 26.7 \\
\hline 35 & 500 & 71 & 4. 1 & 4.5 & .2 & 128 & 5.6 & 114.8 & 1.3 & 22.0 & 24.4 \\
\hline 30 & 560 & 81 & 5.1 & 5.8 & .27 & 134 & 6.2 & 117.3 & 2.3 & 17.8 & 19.6 \\
\hline 25 & 500 & 86 & 6.3 & 6.5 & .19 & 120 & 4.5 & 113.4 & .3 & 15.4 & 15.9 \\
\hline 20 & 570 & 87 & 8.4 & 9.0 & .34 & 121 & 7.2 & 109.2 & .7 & 11.0 & 11.9 \\
\hline 15 & 525 & 71 & 14.6 & 16.2 & .88 & 138 & 9.2 & 116.8 & 1.3 & 6.1 & 6.85 \\
\hline 10 & 456 & 37 & 42.0 & 48.0 & 2.86 & 162 & 13.3 & 126.0 & 1.1 & 2.1 & 2.4 \\
\hline 5 & 250 & 0 & & & & & & & & & \\
\hline
\end{tabular}

Thermal constant of curve: Mean, $129.25^{\circ}$, S. D., 6.7 ${ }^{\circ}$; Ave. Min., $114.3^{\circ}$, S. D., $1.8^{\circ}$. Zero of curve: Mean $6^{\circ}$; Ave. Min., $7^{\circ}$.

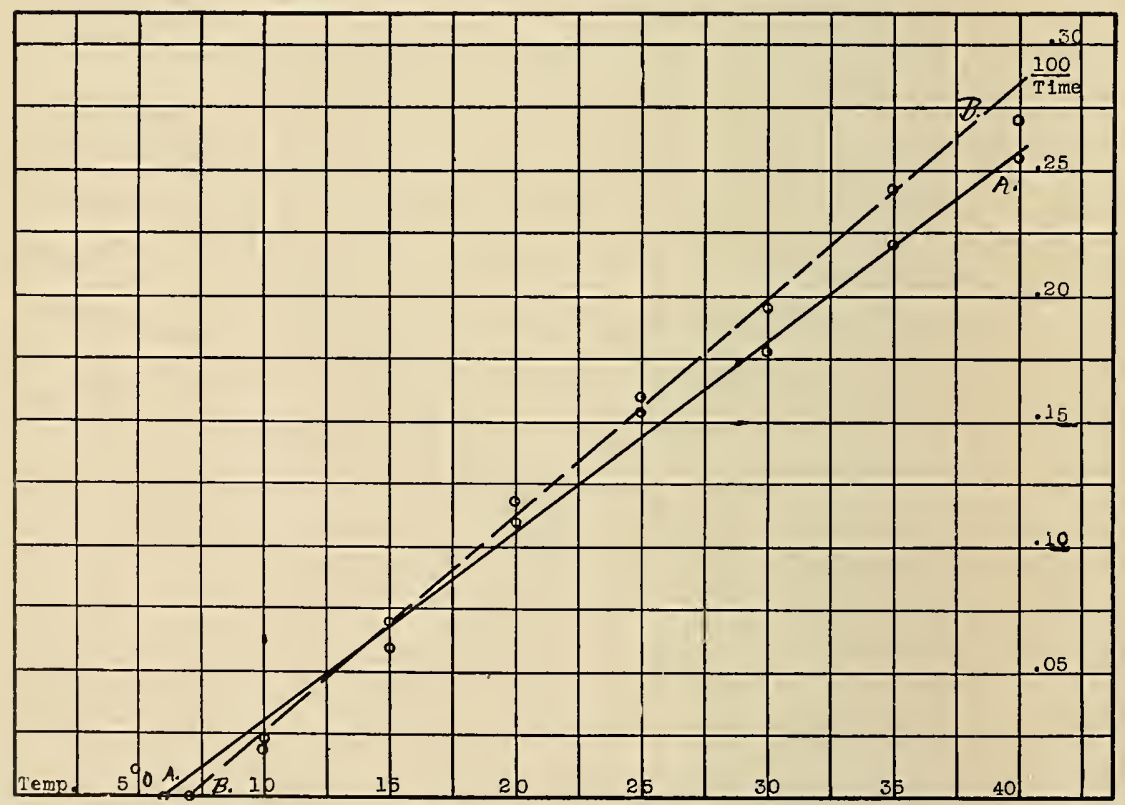

Fig. 8.-Velocity of development of Sarcophaga carnaria (?) pupae. A.-Based upon the time of emergence. B.-Based upon the average time of first emergence for the different lots. (See Table 8) 
TABLE 9.-Chloridea obsoleta, larvae. Constant temperatures (See Fig. 9).

\begin{tabular}{|c|c|c|c|c|c|c|c|c|c|c|c|}
\hline \multirow{2}{*}{ T. } & \multirow{2}{*}{$\begin{array}{l}\text { No. of } \\
\text { Insects }\end{array}$} & \multirow{2}{*}{$\begin{array}{c}\text { Perc't. } \\
\text { Devel- } \\
\text { oped }\end{array}$} & \multicolumn{3}{|c|}{$\begin{array}{l}\text { Length of Stage in } \\
\text { Days }\end{array}$} & \multicolumn{4}{|c|}{ Acc. Effect. Temp. } & \multicolumn{2}{|c|}{ Index of Dev. } \\
\hline & & & Ave. Min. & Mean & S. D. & Mean & S. D. & Min. & S. D. & Mean & Min. \\
\hline $35^{\circ}$ & 100 & 14 & 78 & 8.0 & & $240^{\circ}$ & & $234^{\circ}$ & & 12.5 & 12.8 \\
\hline 30 & 200 & 32 & 90 & 96 & & 240 & & 225 & & 10.4 & 11.1 \\
\hline 25 & 200 & 36 & 114 & 122 & & 245 & & 228 & & 8. 1 & 8.8 \\
\hline 20 & 200 & 40 & 14.2 & 152 & & 229 & & 211 & & 6.5 & 7.0 \\
\hline 15 & 200 & 46 & 210 & 223 & & 224 & & 211 & & 4.4 & 4.7 \\
\hline 10 & 200 & 25 & 48.0 & 530 & & 265 & & 240 & & 2.3 & 2.4 \\
\hline 5 & 100 & 0 & & & & & & & & & \\
\hline
\end{tabular}

Thermal constant of curve: Mean, about $240^{\circ}$; Ave. Min., about $220^{\circ}$. Zero of both curves is $5^{\circ}$ but this is doubtless too low.

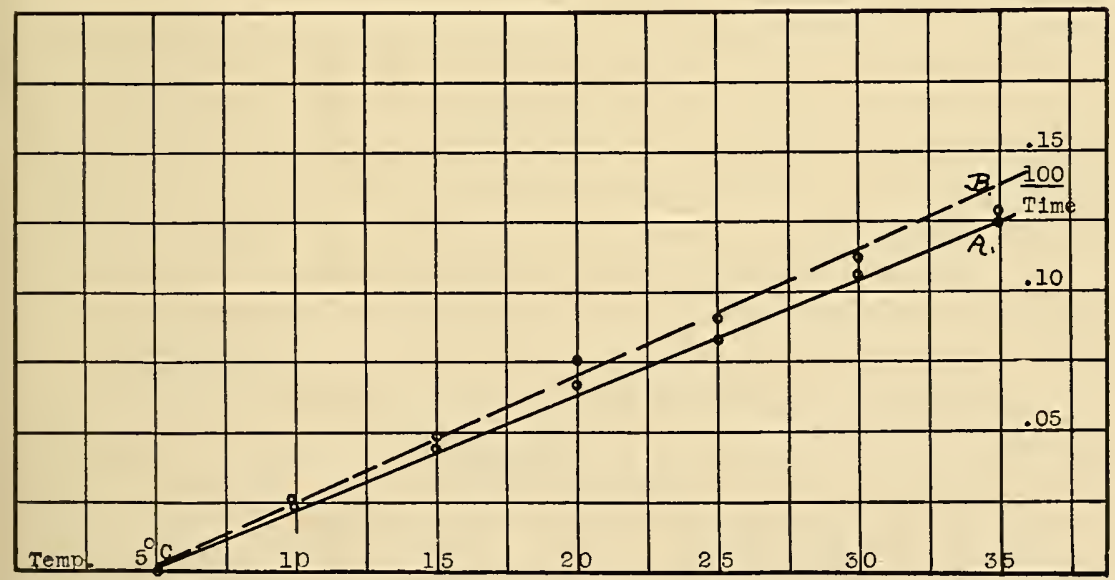

Fig. 9.-Velocity of development of Chloridea obsoleta larvae. A.-Based upon the mean time of pupation. B.-Based upon the average time of first pupation for the different lots. (See Table 9.) 
TABLE 10.-Chloridea obsoleta, pupae. Constant temperatures (See Fig. 10).

\begin{tabular}{|c|c|c|c|c|c|c|c|c|c|c|c|}
\hline \multirow{2}{*}{ T. } & \multirow{2}{*}{$\begin{array}{l}\text { No. of } \\
\text { Insects }\end{array}$} & \multirow{2}{*}{$\begin{array}{l}\text { Perc't. } \\
\text { Devel- } \\
\text { oped }\end{array}$} & \multicolumn{3}{|c|}{ 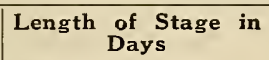 } & \multicolumn{4}{|c|}{ Acc. Effect. Temp. } & \multicolumn{2}{|c|}{ Index of Dev } \\
\hline & & & Ave. Min. & Mean & S. D. & Mean & S. D. & Min. & S. D. & Mean & Min. \\
\hline $35^{\circ}$ & 350 & 9 & 12.0 & 12.5 & .88 & $337.5^{\circ}$ & $23.6^{\circ}$ & $330.0^{\circ}$ & & 8.0 & 8.3 \\
\hline 30 & 350 & 49 & 13.4 & 13.8 & .72 & 301.4 & 22.7 & 301.5 & $8.4^{\circ}$ & 7.2 & 7.5 \\
\hline 25.5 & 350 & 56 & 15.6 & 16.8 & .49 & 294.0 & 8.5 & 288.0 & 6.6 & 6.0 & 6.4 \\
\hline 20 & 400 & 62 & 22.0 & 23.5 & 1.3 & 281.0 & 16.3 & 275.0 & 3.0 & 4.3 & 4.5 \\
\hline 15.5 & 350 & 55 & 32.5 & 39.0 & 3.7 & 292.0 & 27.8 & 260.0 & 5.8 & 2.5 & 3.1 \\
\hline 11 & 350 & 26 & 900 & 980 & 2.2 & 300.0 & 6.6 & 315.0 & 2.0 & 1.0 & 1.1 \\
\hline
\end{tabular}

Thermal constant of curve: Mean, $306^{\circ}$, S. D., 15.4\%; Ave. Min., 295 S. D., $3.8^{\circ}$. Zero of curve: Mean, $8^{\circ}$; Ave. Min., $7.5^{\circ}$.

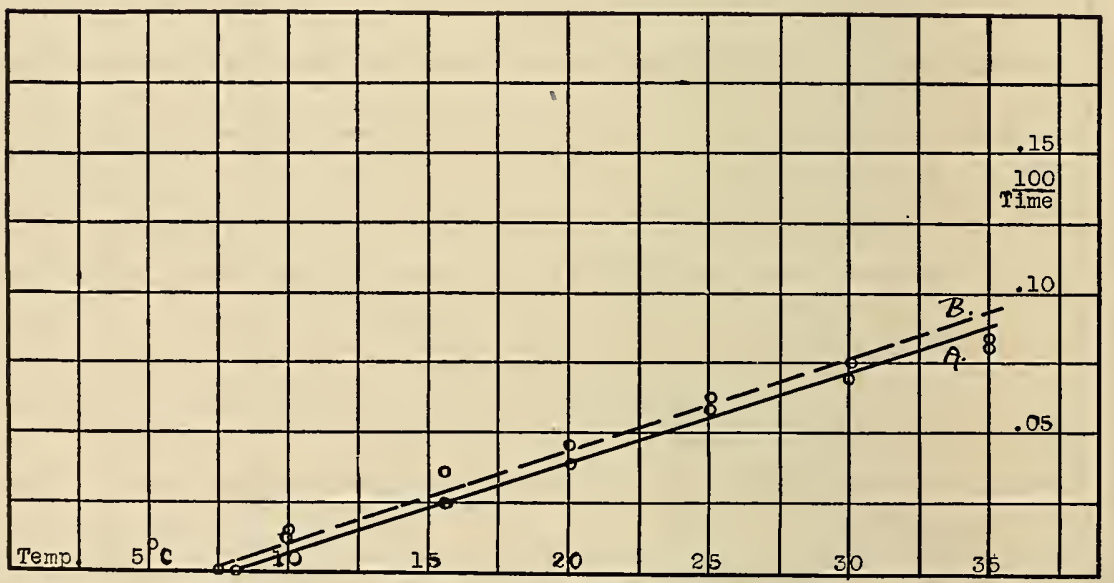

Fig. 10.-Velocity of development of Chloridea obsoleta pupae. A.-Based upon the mean time of emergence. B.-Based upon the average time of first emergence for the different lots. (See Table 10.) 
TABLE 11.-Pontia rapae, larvae. Constant temperatures (See Fig. 11).

\begin{tabular}{|c|c|c|c|c|c|c|c|c|c|c|c|}
\hline \multirow{2}{*}{ T. } & \multirow{2}{*}{$\begin{array}{l}\text { No. of } \\
\text { Insects }\end{array}$} & \multirow{2}{*}{$\begin{array}{c}\text { Perc't. } \\
\text { Devel- } \\
\text { oped }\end{array}$} & \multicolumn{3}{|c|}{$\begin{array}{c}\text { Length of Stage in } \\
\text { Days }\end{array}$} & \multicolumn{4}{|c|}{ Acc. Effect. Temp. } & \multicolumn{2}{|c|}{ Index of Dev. } \\
\hline & & & Ave. Min. & Mean & S. D. & Mean & S. D. & Min. & S. D. & Mean & Min. \\
\hline $35^{\circ}$ & 150 & Larvae & all diec & from & fungus & disease. & & & & & \\
\hline 30 & 200 & 60 & 8.0 & 825 & .62 & $206^{\circ}$ & $154^{\circ}$ & $200^{\circ}$ & $2.0^{\circ}$ & 12.0 & 12.5 \\
\hline 25 & 200 & 69 & 110 & 12.75 & .68 & 242 & 12.8 & 209 & 3.4 & 7.8 & 9.1 \\
\hline 20 & 200 & 80 & 145 & 15.5 & .75 & 217 & 10.4 & 203 & 1.8 & 6.4 & 6.9 \\
\hline 15 & 200 & 58 & 230 & 250 & 98 & 225 & 11.8 & 207 & 7 & 4.0 & 43 \\
\hline 10 & 200 & 46 & 510 & 550 & 2.73 & 220 & 17.3 & 204 & 1.4 & 1.9 & 1.97 \\
\hline 8 & 100 & \multirow{2}{*}{\multicolumn{10}{|c|}{$\begin{array}{l}\text { Larvae all died before pupating. } \\
\text { Larvae did not grow. All died before feeding. }\end{array}$}} \\
\hline 5 & 100 & & & & & & & & & & \\
\hline
\end{tabular}

Thermal constant of curve: Mean, $217^{\circ}$, S. D., $11^{\circ}$; Ave. Min., 205.6 S. D., $2.3^{\circ}$. Zero of curve: Mean, $6^{\circ}$; Ave. Min., $6^{\circ}$.

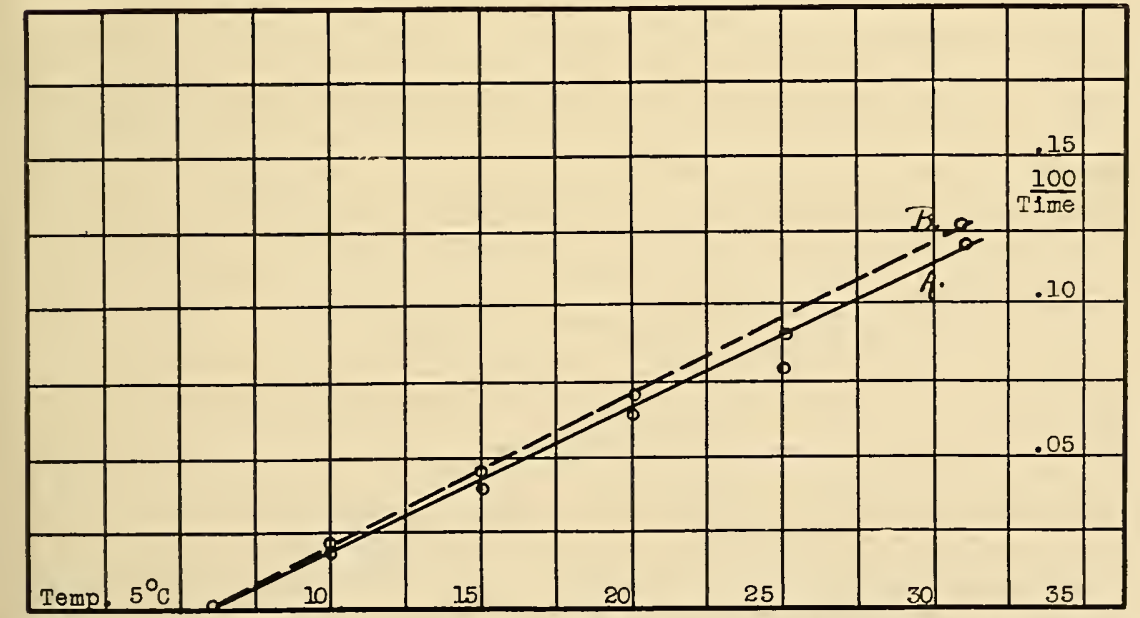

Fig. 11.-Velocity of development of Pontia rapae larvae. A.-Based upon the mean time of pupation. B.-Based upon the average time of first pupation for the different lots. (See Table 11). 
TABLE 12.-Pontia rapae, pupae. Constant temperatures (See Fig. 12).

\begin{tabular}{|c|c|c|c|c|c|c|c|c|c|c|c|c|}
\hline \multirow{2}{*}{$\mathbf{T}$. } & \multirow{2}{*}{$\begin{array}{l}\text { No. of } \\
\text { Insects }\end{array}$} & \multirow{2}{*}{$\begin{array}{c}\text { Perc't. } \\
\text { Devel- } \\
\text { oped }\end{array}$} & \multicolumn{4}{|c|}{$\begin{array}{c}\text { Length of Stage in } \\
\text { Days }\end{array}$} & \multicolumn{4}{|c|}{ Acc. Effect. Temp. } & \multicolumn{2}{|c|}{ Index of Dev. } \\
\hline & & & Ave. Min. & Mean & S. I & D. & Mean & S. D. & Min. & S. D. & Mean & Min. \\
\hline $35^{\circ}$ & 100 & 43 & 5.0 & 5.35 & & .2 & $147^{\circ}$ & $5.5^{\circ}$ & $140.0^{\circ}$ & $2.1^{\circ}$ & 19.0 & 20.0 \\
\hline 30 & 100 & 50 & 5.9 & 6.9 & & .61 & 159 & 14.0 & 135.7 & 3.2 & 14.5 & 16.9 \\
\hline 25 & 100 & 64 & 7.6 & 8.5 & & .57 & 153 & 10.2 & 130.0 & 0 & 11.7 & 125 \\
\hline 20 & 150 & 80 & 10.0 & 11.5 & & .54 & 150 & 7.1 & 130.0 & .2 & 8.7 & 10.0 \\
\hline 15 & 150 & 74 & 17.4 & 180 & & .82 & 144 & 6.6 & 139.2 & .4 & 5.5 & 5.75 \\
\hline 10 & 100 & 39 & 48.0 & 49.0 & & .98 & 147 & 9.0 & 144.0 & 0 & 2.0 & 2.1 \\
\hline 5 & 75 & 0 & & & & & & & & & & \\
\hline
\end{tabular}

Thermal constant of curve: Mean, $150.1^{\circ}$, S. D., $8.2^{\circ}$; Ave. Min., $136.4^{\circ}$, S. D., $4.2^{\circ}$. Zero of curve: Mean, $7^{\circ}$; Ave. Min., $7.5^{\circ}$.

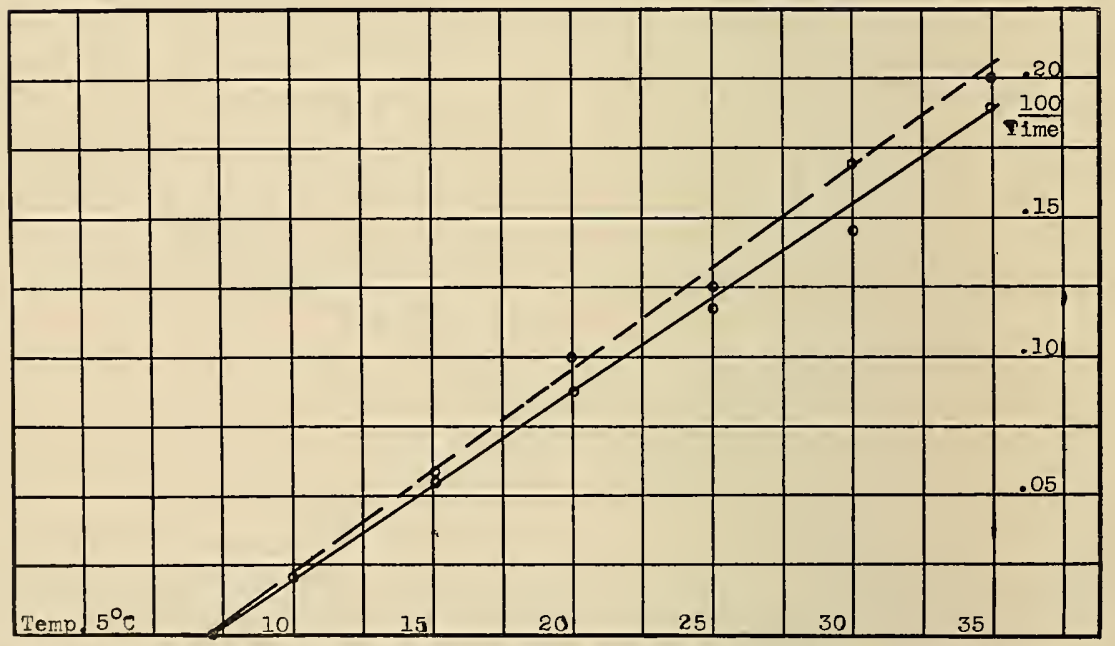

Fig. 12.-Velocity of development of Pontia rapae pupae. A.-Based upon the mean time of emergence. B.-Based upon the average time of first emergence for the different lots. (See Table 12). 
TABLE 13.-Malacosoma americana, pupae. Constant temperatures (See Fig. 13)

\begin{tabular}{|c|c|c|c|c|c|c|c|c|c|c|c|}
\hline \multirow{2}{*}{ T. } & \multirow{2}{*}{$\begin{array}{l}\text { No. of } \\
\text { Insects }\end{array}$} & \multirow{2}{*}{$\begin{array}{c}\text { Perc't. } \\
\text { Devel- } \\
\text { oped }\end{array}$} & \multicolumn{3}{|c|}{$\begin{array}{c}\text { Length of Stage in } \\
\text { Days }\end{array}$} & \multicolumn{4}{|c|}{ Acc. Effect. Temp. } & \multicolumn{2}{|c|}{ Index of Deve } \\
\hline & & & Ave. Min. & Mean & S. D. & Mean & S. D. & Min. & S. D. & Mean & Min. \\
\hline $35^{\circ}$ & 50 & 58 & 8.5 & 9.5 & .33 & $239.2^{\circ}$ & $37^{\circ}$ & $\left|233.7^{\circ}\right|$ & $0^{\circ}$ & 10.5 & 11.7 \\
\hline 30 & 100 & 42 & 10.5 & 11.3 & .55 & 237.0 & 4.8 & 236.3 & 0 & 8.8 & 9.5 \\
\hline 25 & 100 & 64 & 14.0 & 15.1 & .62 & 248.0 & 10.2 & 245.0 & 0 & 6.5 & 7.1 \\
\hline 20 & 100 & 70 & 18.8 & 225 & .81 & 247.0 & 8.9 & 235.0 & 1.6 & 4.4 & 5.3 \\
\hline 15 & 100 & 33 & 34.0 & 47.0 & 3.63 & 282.0 & 21.7 & 255.0 & 0 & 2.1 & 2.9 \\
\hline 10 & 50 & No & emerger & & & & & & & & \\
\hline
\end{tabular}

Thermal constant of curve: Mean, $247^{\circ}$, S. D., $11.2^{\circ}$; Ave. Min., $239^{\circ}$, S. D., $7.4^{\circ}$. Zero of curve: Mean, $9^{\circ}$; Ave. Min., $7.5^{\circ}$.

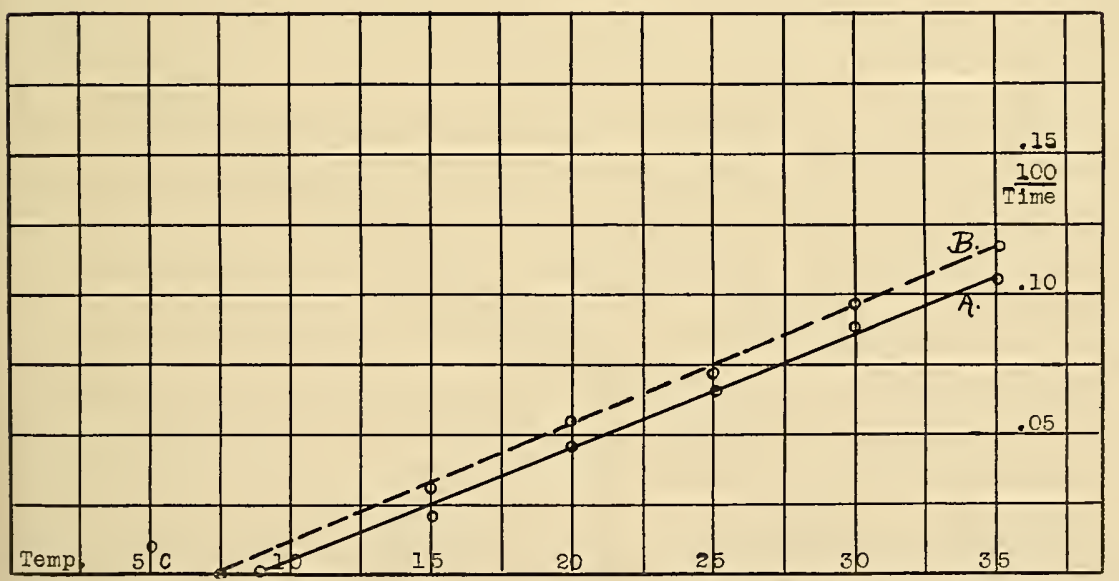

Fig. 13.-Velocity of development of Malacosoma americana pupae. A.-Based upon the mean time of emergence. B.-Based upon the average time of first emergence for the different lots. (See Table 13). 
TABLE 14.-Carpocapsa pomonella; pupae. Constant temperatures (See Fig. 14)

\begin{tabular}{|c|c|c|c|c|c|c|c|c|c|c|c|}
\hline \multirow{2}{*}{$\mathbf{T}$} & \multirow{2}{*}{$\begin{array}{l}\text { No. of } \\
\text { Insects }\end{array}$} & \multirow{2}{*}{$\begin{array}{c}\text { oped } \\
\text { Perc't. } \\
\text { Devel- }\end{array}$} & \multicolumn{3}{|c|}{$\begin{array}{c}\text { Length of Stage in } \\
\text { Days }\end{array}$} & \multicolumn{4}{|c|}{ Acc. Effect. Temp. } & \multicolumn{2}{|c|}{ Index of Dev. } \\
\hline & & & Ave. Min. & Mean & S. D. & Mean & S. D. ! & Min. & S. D. & Mean & Min. \\
\hline $35^{\circ}$ & 75 & 73 & 6.0 & $\begin{array}{ll}6 & 25\end{array}$ & .54 & $162.5^{\circ}$ & $14.0^{\circ}$ & $159.0^{\circ}$ & & 16.0 & 167 \\
\hline 30 & 90 & 75 & 7.0 & 725 & .37 & 152.7 & 8.5 & 150.5 & $1.3^{\circ}$ & 13.8 & 14.3 \\
\hline 25 & 90 & 82 & 84 & 88 & .44 & 1408 & 7.0 & 138.3 & 2.1 & 11.4 & 11.9 \\
\hline 20 & 100 & 78 & 12.2 & 133 & .79 & 146.0 & 8.6 & 140.3 & 3.0 & 7.5 & 82 \\
\hline 15 & 100 & 70 & 22.0 & 260 & 1.12 & 156.0 & 11.3 & 143.0 & 4.2 & 3.8 & 45 \\
\hline 12 & 50 & 36 & 440 & 510 & 2. 19 & 153.0 & 6.6 & 154.0 & 0 & 2.0 & 23 \\
\hline
\end{tabular}

Thermal constant of curve: Mean, $150^{\circ}$, S. D., $8.9^{\circ}$; Ave. Min., $145.6^{\circ}$, S. D., $7.8^{\circ}$. Zero of curve: Mean, $9^{\circ}$; Ave. Min., $8.5^{\circ}$.

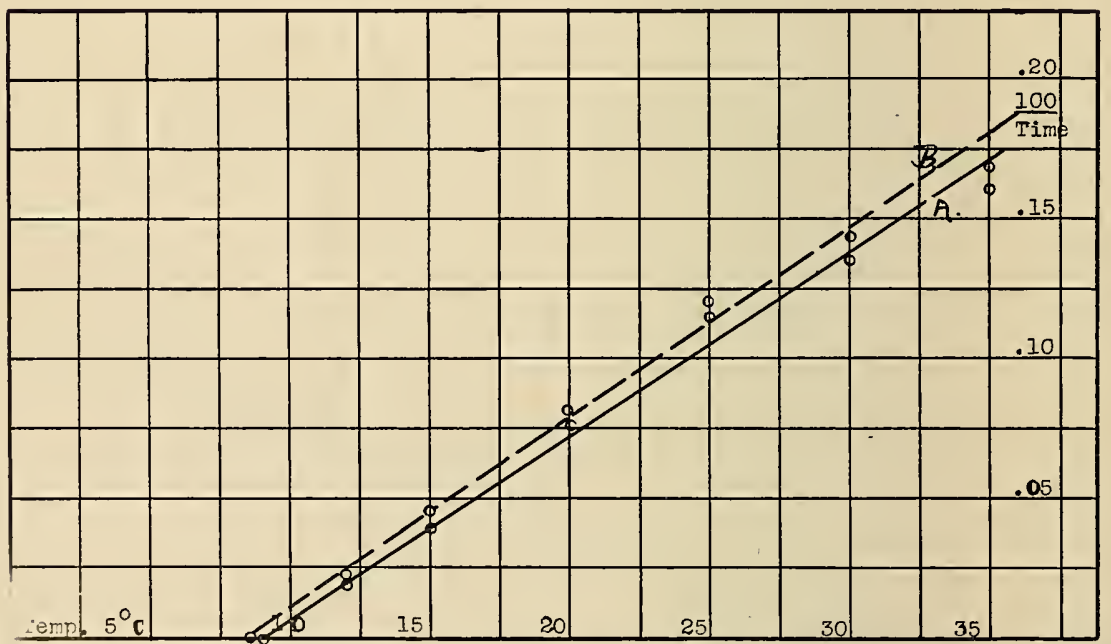

Fig. 14.-Velocity of development of Carpocapsa pomonella pupae. A.-Based upon the mean time of emergence. B.-Based upon the average time of first emergence for the different lots. (See Table 14). 
TABLE 15.-Leptinotarsa 10-lineata; eggs. Constant temperatures (See Fig. 15).

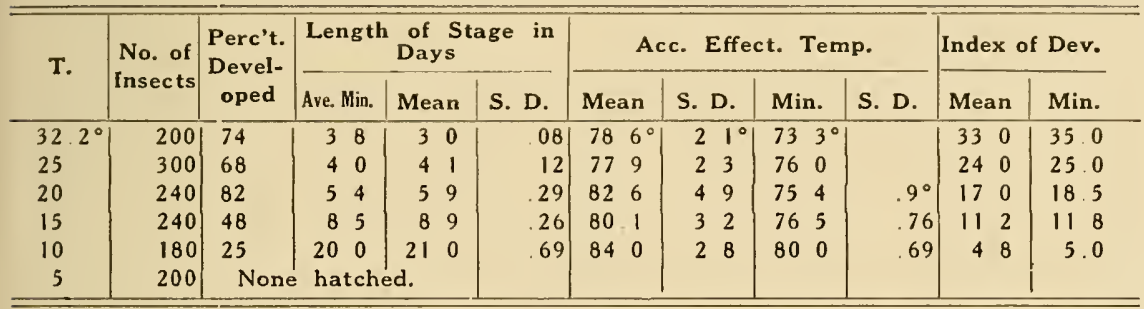

Thermal constant of curve: Mean, $79^{\circ}$, S. D., $2.9^{\circ}$; Ave. Min., 76.8 ${ }^{\circ}$, S. D., $.29^{\circ}$. Zero of curve: Mean, $6^{\circ}$; Ave. Min., $6^{\circ}$.

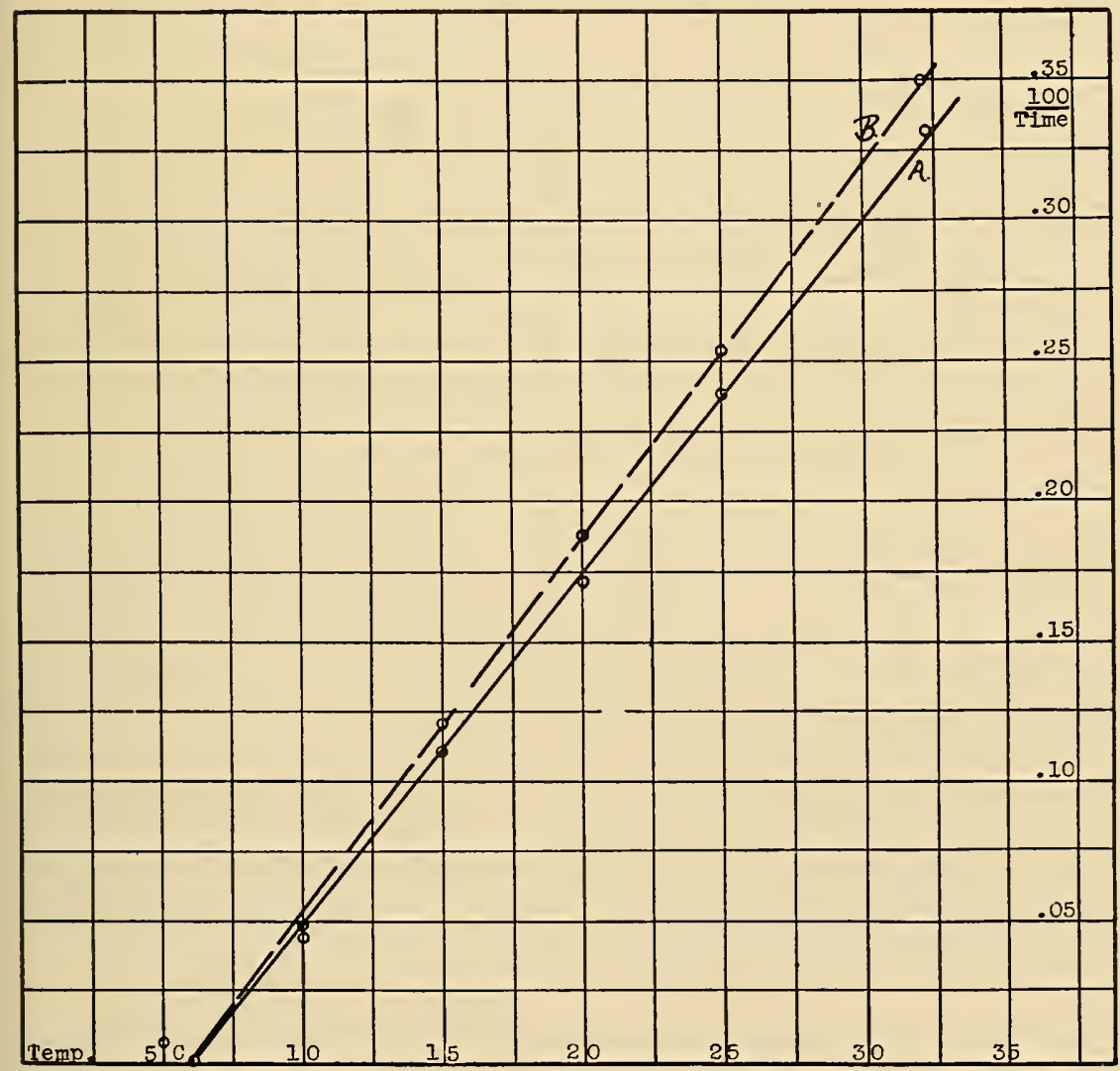

Fig. 15.-Velocity of development of Leptinotarsa 10-lineata eggs. A.-Based upon the mean time of hatching. B.-Based upon the average time of first hatching for the different lots. (See Table 15.) 
TABLE 16. - Leptinotarsa 10-lineata, larvae. Constant temperatures (See Fig. 16)

\begin{tabular}{|c|c|c|c|c|c|c|c|c|c|c|c|}
\hline \multirow{2}{*}{ T. } & \multirow{2}{*}{$\begin{array}{l}\text { No. of } \\
\text { Insects }\end{array}$} & \multirow{2}{*}{$\begin{array}{c}\text { Perc't. } \\
\text { Devel- } \\
\text { oped }\end{array}$} & \multicolumn{3}{|c|}{$\begin{array}{c}\text { Length } \begin{array}{c}\text { of Stage in } \\
\text { Days }\end{array} \\
\text { Says }\end{array}$} & \multicolumn{4}{|c|}{ Acc. Effect. Temp. } & \multicolumn{2}{|c|}{ Index of Dev } \\
\hline & & & Ave. Min. & Mean & S. D. & Mean & S. D. & Min. & S. D. & Mean & Min. \\
\hline $35^{\circ}$ & 200 & Only & four & oupated. & & & & & & & \\
\hline 30 & 200 & 52 & 7.8 & 9.5 & .81 & $237^{\circ}$ & $20.0^{\circ}$ & $188.8^{\circ}$ & $4.7^{\circ}$ & 10.5 & 12.8 \\
\hline 25 & 250 & 60 & 10.0 & 11.75 & .73 & 235 & 14.6 & 192.0 & & 8.5 & 10.0 \\
\hline 20 & 250 & 71 & 13.3 & 15.0 & .56 & 225 & 9.9 & 188.9 & 2.6 & 6.7 & 7.5 \\
\hline 15 & 240 & 67 & 20.0 & 20.25 & .96 & 202 & 18.2 & 184.0 & .7 & 4.8 & 5.0 \\
\hline 10 & 250 & 38 & 45.5 & 46.5 & 2.8 & 232 & 13.9 & 191.1 & 1.1 & 2.2 & 2.2 \\
\hline
\end{tabular}

Thermal constant of curve: Mean, $230^{\circ}$, S. D., $12.6^{\circ}$; Ave. Min., $189.6^{\circ}$, S. D., $8^{\circ}$. Zero of curve: Mean, $5^{\circ}$; Ave. Min., $5.8^{\circ}$.

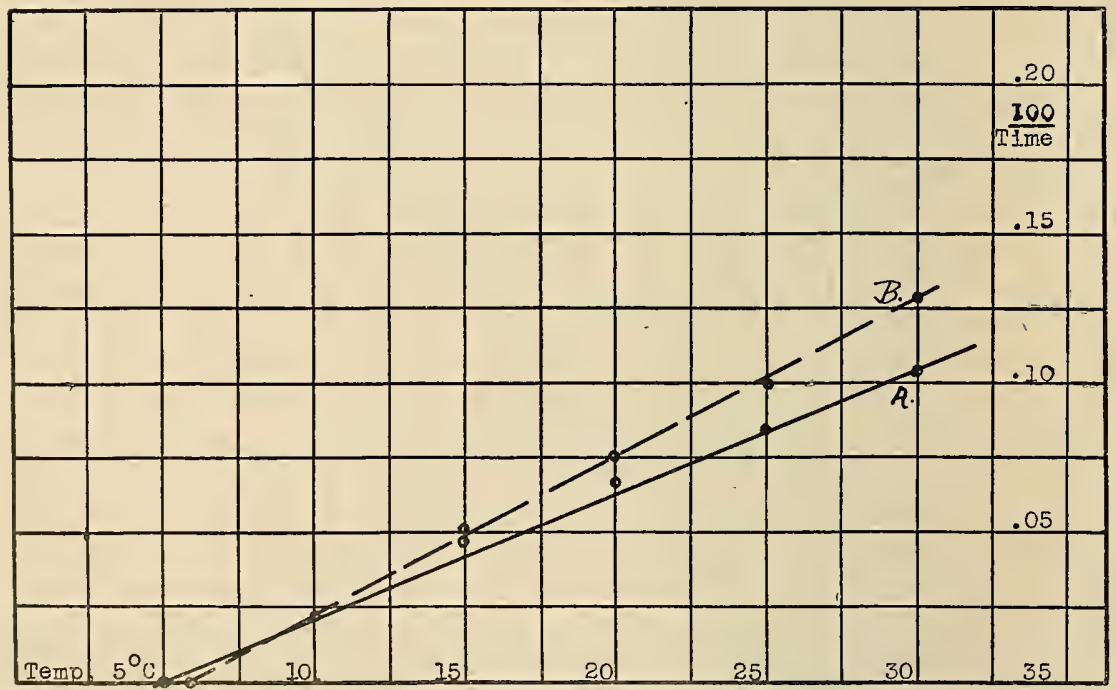

Fig. 16.-Velocity of development of Leptinotarsa 10-lineate larvae. A.-Based upon the mean time of pupation. B.-Based upon the average time of first pupation for the different lots. (See Table 16.) 
TABLE 17.-Leptinotarsa 10-lineata, pupae. Constant temperatures (See Fig. 17)

\begin{tabular}{|c|c|c|c|c|c|c|c|c|c|c|c|}
\hline \multirow{2}{*}{ T. } & \multirow{2}{*}{$\begin{array}{l}\text { No. of } \\
\text { Insects }\end{array}$} & \multirow{2}{*}{$\begin{array}{c}\text { Perc't. } \\
\text { Devel- } \\
\text { oped }\end{array}$} & \multicolumn{3}{|c|}{$\underset{\text { Length of Stage in }}{\text { Days }}$} & \multicolumn{4}{|c|}{ Acc. Effect. Temp. } & \multicolumn{2}{|c|}{ Index of Dev. } \\
\hline & & & Ave. Min. & Mean & S. D. & Mean & S. D. & Min. & S. D. & Mean & Min. \\
\hline $35^{\circ}$ & 75 & 14 & 4.5 & 4.5 & & $1123.7^{\circ}$ & & $123.7^{\circ}$ & & 22.0 & 22.0 \\
\hline 30 & 100 & 47 & 45 & 48 & .22 & 108.0 & $49^{\circ}$ & 101.2 & & 21.7 & 22.0 \\
\hline 25 & 100 & 61 & 55 & 625 & .13 & 109.4 & 22 & 96.2 & & 160 & 181 \\
\hline 20 & 100 & 70 & 80 & 86 & .24 & 107.5 & 30 & 100.0 & & 11.6 & 12.5 \\
\hline 15 & 95 & 78 & 135 & 140 & .42 & 105.0 & 32 & 101.2 & & 71 & 74 \\
\hline 10 & 100 & 40 & 460 & 50.0 & 2.37 & 125.0 & 6.0 & 115.0 & & 2.0 & 2.2 \\
\hline
\end{tabular}

Thermal constant of curve: Mean, $109.9^{\circ}$, S. D., $3.6^{\circ}$; Ave. Min., $102.6^{\circ}$, S. D., $1.4^{\circ}$. Zero of curve: Mean, $7.5^{\circ}$; Min. Ave., $7.5^{\circ}$.

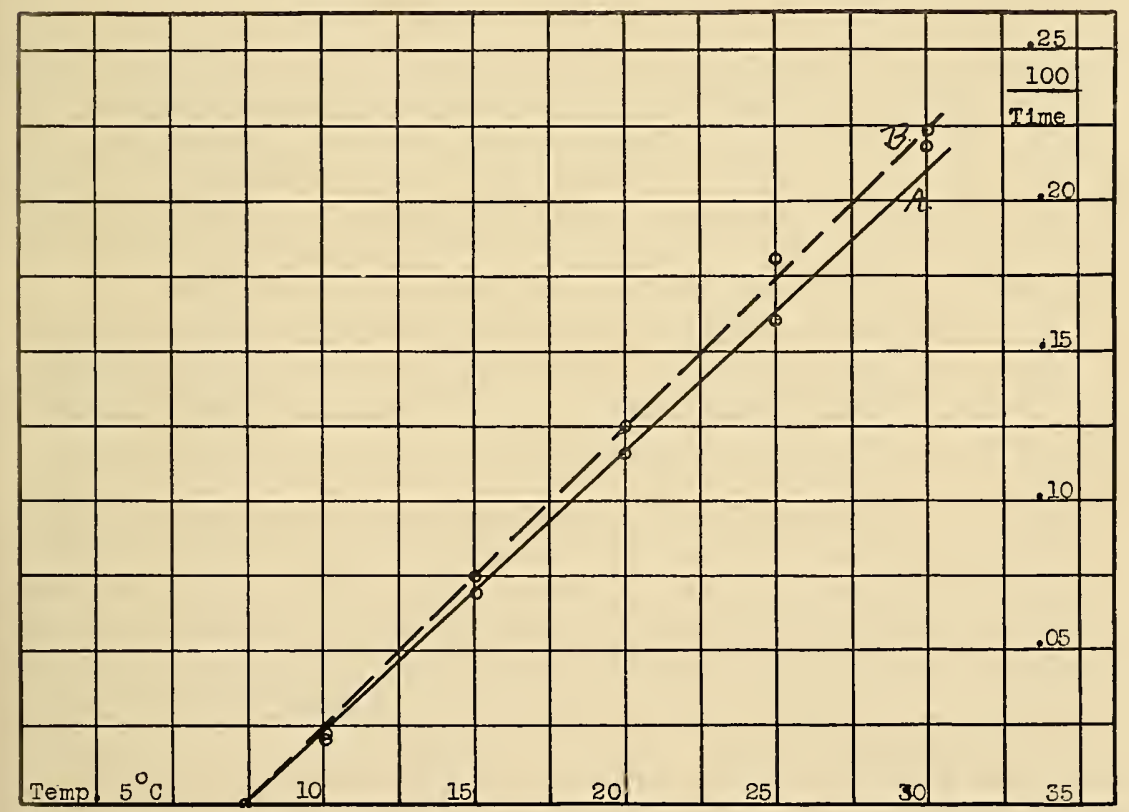

Fig. 17.-Velocity of development of Leptinotarsa 10-lineate pupae. A.-Based upon the mean time of emergence. B.-Based upon the average time of first emergence for the different lots. (See Table 17.) 


\section{THE THRESHOLD OF DEVELOPMENT}

If it is true that there is, at temperatures sufficiently low, a definite cessation of development there must be some point at which the developmental processes no longer operate or at which development, arrested by low temperatures, again begins to manifest itself when temperatures are gradually raised.

The determination of the location of this point, or threshold of development, has been the object of a supplementary series of experiments. It was found that development did not occur, or did not continue to completion, at temperatures, in the constant temperature series, which approached to within one to five degrees of the zero point of the velocity curves. Observations of larvae showed that they would usually feed and grow to some extent, for a few days, at temperatures often within one degree of the zero of the velocity curve.

No perceptible growth of newly hatched larvae and no feeding of such larvae could ever be detected at this zero point although well grown larvae, transferred from higher temperatures, apparently fed to a slight extent but accomplished no preceptible growth. Young larvae, at the zero point, usually died within a few hours; older larvae lived for a more or less indefinite period, possibly as long as a month, but no perceptible growth ever occurred.

At one or two degrees above this zero point larvae, ready to pupate, transferred from warm incubators, would sometimes pupate. Larvae, therefore, showed some developmental activity at temperatures close to the zero of the velocity curve. The lack of evidence of development at that point was taken to indicate that it could not be much above the actual threshold while evidence of some development almost at the zero indicated that it was not below the threshold. Since developmental processes were never completed at these temperatures, the time factor could not be measured to determine whether it would coincide with the point indicated by the velocity curve.

Development of pupae, when incomplete, is not evidenced by any external change. Internal changes are of such a nature in the early period of pupal development as to afford little evidence of slight development. Experiments designed to determine possible development of pupae at temperatures down to the zero of the velocity curves were arranged, based upon the assumption that some development might be possible at low temperatures even though complete transformation was not. These experiments con- 
sisted of placing different lots of pupae in low temperature incubators for periods and transferring them to warmer temperatures to complete their development.

It was thought that if measurable development had taken place at the low temperature it should have manifested itself in the reduction of the time necessary to complete the transformation at the higher temperature. In these tests a factor which apparently affects some of the results in the constant temperature series is again of importance. This is an apparent loss of vitality incident to the prolonged vital period, possibly connected with the expenditure of energy represented by the basal metabolism indicated in the carbon dioxide tests, and a change in the physical condition of the pupal covering which seems to be in the nature of a ripening which possibly renders escape from the puparium, by the fully developed fly, more than normally difficult. These possible factors are discussed in connection with the constant temperature series on pages 13 and 14 .

TABLE 18.-Development of pupae of $L$. caesar kept first in cold temperature incubators at near the zero of the curve of velocity of development $\left(6^{\circ}\right.$ to $6.5^{\circ}$ ) and removed, after varying periods, to incubator at $20^{\circ}$.

\begin{tabular}{|c|c|c|c|c|c|c|}
\hline $\begin{array}{l}\text { No. of } \\
\text { Insects }\end{array}$ & $\begin{array}{c}\text { Days at } \\
6-6.5^{\circ}\end{array}$ & $\begin{array}{l}\text { Acc. } \\
\text { Temp. }\end{array}$ & $\begin{array}{l}\text { Days at } 20^{\circ} \\
\text { (Minimum) }\end{array}$ & Acc. Temp. & $\begin{array}{c}\text { Total } \\
\text { Acc. Temp. }\end{array}$ & \begin{tabular}{|c} 
Excess Over \\
$122.8^{\circ}$
\end{tabular} \\
\hline 200 & $30^{\circ}$ & $0^{\circ}$ & 94 & $127^{\circ}$ & $127^{\circ}$ & $4.2^{\circ}$ \\
\hline 100 & 60 & 0 & 93 & 126 & 126 & 3.2 \\
\hline 200 & 90 & 0 & 94 & 127 & 127 & 4.2 \\
\hline 200 & 130 & 0 & 96 & 1296 & 129.6 & 6.8 \\
\hline 200 & 180 & 0 & 10 & 135 & 135 & 12.2 \\
\hline 200 & 235 & 0 & 105 & 142 & 142 & 19.2 \\
\hline 200 & 272 & 0 & 104 & 141 & 141 & 18.2 \\
\hline 140 & 335 & 0 & 11 & 1485 & 148.5 & 25.7 \\
\hline 200 & 366 & 0 & 115 & 155 & 155 & 32.2 \\
\hline 200 & 418 & 0 & 12 & 162 & 162 & 39.2 \\
\hline 200 & 480 & 0 & 125 & 108.7 & 168.7 & 45.9 \\
\hline 200 & 540 & 0 & 12 & 162 & 162 & 39.2 \\
\hline 300 & 611 & 0 & 15 & 2025 & 202.5 & 79.2 \\
\hline 300 & 654 & 0 & 13 & $175 \quad 5$ & 175.5 & 52.7 \\
\hline 284 & 691 & 0 & 14 & 189 & 189 & 66.2 \\
\hline
\end{tabular}

Thermal constant for the insect as indicated by the velocity curve-122.8 , S. D. $9.2^{\circ}$, based on mean emergence.

Emergence varied from less than 1 to 31 per cent in all lots in the cold conditions longer than 90 days. Two individuals emerged from the last lot. All were deformed in some manner and were unable to fly.

A second similar series with the blowfly gave results as shown in Table 19. Accidental warming of the cold storage chamber spoiled this material so the test was abandoned.

Tables 18 and 19 indicate that there is no development accomplished at the temperatures equivalent to those indicated as zero 
TABLE 19.-Pupae of blowfly in incubator at the zero indicated by the velocity curve, $6.5^{\circ}$ to $7^{\circ}$; transferred to the $20^{\circ}$ incubator.

\begin{tabular}{|c|c|c|c|c|}
\hline $\begin{array}{l}\text { No. of } \\
\text { Insects }\end{array}$ & $\begin{array}{l}\text { Days at } \\
6,5-7^{\circ}\end{array}$ & $\begin{array}{c}\text { Days at } 20^{\circ} \\
\text { (Minimum) }\end{array}$ & $\begin{array}{l}\text { Effect. Temp. } \\
\text { Accumulated }\end{array}$ & $\begin{array}{c}\text { Excess Over } \\
130.1^{\circ}\end{array}$ \\
\hline 100 & 30 & 10.3 & $133.9^{\circ}$ & $3.8^{\circ}$ \\
\hline 200 & 90 & 12 & 156 & 25.9 \\
\hline 200 & 180 & 12.6 & 164 & 33.9 \\
\hline
\end{tabular}

Thermal constant indicated by the velocity curve $130.1^{\circ}, \mathrm{S}$. D., $5.7^{\circ}$.

TABLE 20.-Development of pupae of the blowfly incubated first at $8^{\circ}$ then at $20^{\circ}$.

\begin{tabular}{c|c|c|c|c|c|c}
\hline $\begin{array}{c}\text { No. of } \\
\text { Pupae }\end{array}$ & $\begin{array}{c}\text { Days at } \\
\mathbf{8 0}^{\circ}\end{array}$ & $\begin{array}{c}\text { Acc. Effect. } \\
\text { Temp. at } \mathbf{8}^{\circ}\end{array}$ & $\begin{array}{c}\text { Days at } \\
\mathbf{2 0}^{\circ}\end{array}$ & $\begin{array}{c}\text { Acc. Effect. } \\
\text { Temp. at 200 }\end{array}$ & $\begin{array}{c}\text { Total Effect. } \\
\text { Temp. }\end{array}$ & $\begin{array}{c}\text { Apparent } \\
\text { Effective } \\
\text { Temp. at 8 }\end{array}$ \\
\hline 200 & 42 & $42^{\circ}$ & 7.2 & $93.6^{\circ}$ & $135.6^{\circ}$ & $36.5^{\circ}$ \\
200 & 60 & 60 & 5.8 & 75.4 & 1354 & 54.7 \\
200 & 70 & 70 & 5.2 & 67.6 & 137.6 & 62.5 \\
200 & 80 & 80 & 5.3 & 68.9 & 148.9 & 61.2 \\
200 & 90 & 90 & 5.0 & 65.0 & 145.0 & 650 \\
200 & 120 & 120 & 6.0 & 78.0 & 198.0 & 52.0 \\
200 & 150 & 150 & 7.5 & 101.4 & 251.4 & 597 \\
\hline
\end{tabular}

Zero of velocity curve, $7^{\circ}$; Thermal constant indicated by curve, $130.1^{\circ}$, S. D., 5.7.

TABLE 21.-Development of pupae of Lucilia caesar kept first at $7^{\circ}$ and later transferred to $20^{\circ}$ incubator.

\begin{tabular}{c|c|c|c|c|c|c}
\hline $\begin{array}{c}\text { No. of } \\
\text { Pupae }\end{array}$ & $\begin{array}{c}\text { Days at } \\
7^{\circ}\end{array}$ & $\begin{array}{c}\text { Acc. Effect. } \\
\text { Temp. at 70 }\end{array}$ & $\begin{array}{c}\text { Days at } \\
20^{\circ}\end{array}$ & $\begin{array}{c}\text { Acc. Effect. } \\
\text { Temp. at 20 }\end{array}$ & $\begin{array}{c}\text { Total Effect. } \\
\text { Temp. }\end{array}$ & $\begin{array}{c}\text { Apparent } \\
\text { Effective } \\
\text { Temp. at 70 }\end{array}$ \\
\hline 500 & 15 & $7.5^{\circ}$ & 8.2 & $106.7^{\circ}$ & $114.2^{\circ}$ & $18.6^{\circ}$ \\
500 & 30 & 15.0 & 8.4 & 109.4 & 124.4 & 13.4 \\
500 & 60 & 30.0 & 7.8 & 101.3 & 131.3 & 21.5 \\
500 & 90 & 45.0 & 9.0 & 121.5 & 166.5 & 1.3 \\
500 & 120 & 60.0 & 57 & 113.0 & 173.0 & 9.8 \\
\hline
\end{tabular}

Zero of velocity curve $6.5^{\circ}$; Thermal constant indicated by curve $122.8^{\circ}$, S. D., $9.2^{\circ}$.

TABLE 22.-Development of tent-caterpillar pupae incubated first at $10^{\circ}$ then transferred to $20^{\circ}$ incubator.

\begin{tabular}{c|c|c|c|c|c|c}
\hline $\begin{array}{c}\text { No. of } \\
\text { Pupae }\end{array}$ & $\begin{array}{c}\text { Days at } \\
10^{\circ}\end{array}$ & $\begin{array}{c}\text { Acc. Effect. } \\
\text { Temp. at 10 }\end{array}$ & $\begin{array}{c}\text { Days at } \\
\mathbf{2 0}^{\circ}\end{array}$ & $\begin{array}{c}\text { Acc. Effect. } \\
\text { Temp. at 20 }\end{array}$ & $\begin{array}{c}\text { Total Effect. } \\
\text { Temp. }\end{array}$ & $\begin{array}{c}\text { Apparent } \\
\text { Effective } \\
\text { Temp. at 100 }\end{array}$ \\
\hline 20 & 30 & $30^{\circ}$ & 21 & $231^{\circ}$ & $261^{\circ}$ & $16^{\circ}$ \\
20 & 45 & 45 & 21 & 231 & 276 & 16 \\
20 & 75 & 75 & 20 & 220 & 295 & 25 \\
20 & 120 & 120 & 22 & 242 & 362 & 5 \\
\hline
\end{tabular}

Zero of velocity curve $9^{\circ}$; Thermal constant indicated by the curve $247^{\circ}$, S. D., $11.2^{\circ}$. 
TABLE 23.-Development of pupae of the blowfly incubated first at $20^{\circ}$ and then transferred to incubator at $8^{\circ}$.

\begin{tabular}{c|c|c|c|c|c|c}
$\begin{array}{c}\text { No. of } \\
\text { Pupae }\end{array}$ & $\begin{array}{c}\text { Days at } \\
20^{\circ}\end{array}$ & $\begin{array}{c}\text { Acc. Effect. } \\
\text { Temp. at 20 }\end{array}$ & $\begin{array}{c}\text { Days at } \\
8^{\circ}\end{array}$ & $\begin{array}{c}\text { Acc. Effect. } \\
\text { Temp. at } 8^{\circ}\end{array}$ & $\begin{array}{c}\text { Total Effect. } \\
\text { Temp. }\end{array}$ & $\begin{array}{c}\text { Apparent } \\
\text { Effective } \\
\text { Temp. at 8 }\end{array}$ \\
\hline 100 & 8 & $104^{\circ}$ & 23 & $23^{\circ}$ & $127^{\circ}$ & $26.1^{\circ}$ \\
100 & 7 & 91 & 46 & 46 & 137 & 39.1 \\
100 & 6 & 78 & No emergence after 90 days when observations were \\
discontinued.
\end{tabular}

Zero indicated by velocity curve $7^{\circ}$; Thermal constant $130.1^{\circ}$; S. D., $5.7^{\circ}$.

points by the curves of velocity of development, or that any development which may take place is not sufficient to overcome the effect of the retardation incident to the undue prolongation of the developmental stage.

It is, of course, possible that slight development may have taken place at these or even at lower temperatures, but escaped observation.

Tables 20 to 23 , inclusive, show, on the other hand, that there is perceptible development at temperatures from one-half degree to one degree above the zero indicated by the velocity curve. In one or two cases this development is slightly greater than was to have been expected if the zero of the curve represents the true threshold of development. (See Lot 1, Table 21, and Lot 1, Table 23). Usually the infiuence of the low temperature exposures is rather less than their time would indicate. Generally, when the time was not prolonged to more than about 60 days, the departure from the thermal constant was but little, if any, greater than the deviation from the constant temperature data.

\section{Variable Temperatures}

To determine any possible effect arising from the mere fact of variation in temperatures, and to determine also whether temperature summations based on ordinary outdoor conditions are comparable to those based on constant temperatures, two series of experiments were conducted. The one was merely the recording of the time of development of insects under outdoor conditions, the temperature being measured with a thermograph and the thermal increment, calculated from the zero of the constant temperature velocity curves, compared with that derived from equivalent constant temperature conditions. The second series consisted of a shifting of material from one constant temperature incubator to others, so as to get an effect comparable to a daily variation which might occur outside and a comparison of the data secured 
from this, with the outdoor series and with constant temperature series. This should show whether any variation noted in the behavior of the insects reared outdoors is due to the variation in temperature or to some unrecognized factor.

TABLE 24.-Development of insects under outdoor conditions. Accumulated temperatures above zero of the velocity curves measured from thermograph charts with a planimeter.

\begin{tabular}{|c|c|c|c|c|c|c|c|c|c|}
\hline \multirow[t]{2}{*}{ Insect } & \multirow[t]{2}{*}{ Stage } & \multirow{2}{*}{$\begin{array}{l}\text { Zero of } \\
\text { Curves }\end{array}$} & \multirow{2}{*}{$\begin{array}{l}\text { Therm. } \\
\text { Const. }\end{array}$} & \multirow{2}{*}{$\begin{array}{l}\text { Mean } \\
\text { Temp. }\end{array}$} & \multirow{2}{*}{$\begin{array}{l}\text { Time } \\
\text { Days }\end{array}$} & \multirow{2}{*}{$\begin{array}{l}\text { Accumu- } \\
\text { lated } \\
\text { Temp. }\end{array}$} & \multirow{2}{*}{$\begin{array}{l}\text { Calculated Time } \\
\text { at Constant } \\
\text { Temp. (Days) }\end{array}$} & \multicolumn{2}{|c|}{$\begin{array}{l}\text { Variation from } \\
\text { Therm. Const. }\end{array}$} \\
\hline & & & & & & & & Plus & Minus \\
\hline L. caesar & pupae & $65^{\circ}$ & $122.8^{\circ}$ & $212^{\circ}$ & 82 & $1205^{\circ}$ & 835 & & $2.3^{\circ}$ \\
\hline L. caesar & larvae & 55 & 110.7 & 209 & 64 & 98.6 & 72 & & 12.1 \\
\hline C. vomitoria & pupae & 70 & 130.1 & 212 & 8.4 & 120.3 & 92 & & 9.8 \\
\hline C. vomitoria & larvae & 60 & 104.7 & 205 & 66 & 947 & 72 & & 10.0 \\
\hline L. 10-lineata & egg & 60 & 700 & 20.4 & 51 & 73.4 & 55 & & 5.6 \\
\hline
\end{tabular}

TABLE 25.-Development of blowfly at different constant temperatures. Temperature constant at one point for one or more days then changed to a different constant for the remainder of the period of development.

\begin{tabular}{|c|c|c|c|c|c|c|}
\hline \multirow{2}{*}{$\begin{array}{c}\text { First } \\
\text { Temp. }\end{array}$} & \multirow{2}{*}{$\begin{array}{c}\text { Time at } \\
\text { First } T . \\
\text { (Days) }\end{array}$} & \multirow{2}{*}{$\begin{array}{c}\text { Second } \\
\text { Temp. }\end{array}$} & \multirow{2}{*}{$\begin{array}{c}\text { Time at } \\
\text { Second T. } \\
\text { (Days) }\end{array}$} & \multirow{2}{*}{$\begin{array}{c}\text { Accum. } \\
\text { Temp. }\end{array}$} & Variation from & \multirow{2}{*}{$\begin{array}{l}\text { Constant } \\
\text { Minus }\end{array}$} \\
\hline & & & & & Plus & \\
\hline $30^{\circ}$ & 2 & $10^{\circ}$ & 148 & $1072^{\circ}$ & $2.5^{\circ}$ & \\
\hline 20 & 2 & 10 & 18.25 & 101.0 & & $3.7^{\circ}$ \\
\hline 10 & 2 & 30 & 43 & 1112 & 6.5 & \\
\hline 10 & 2 & 20 & 6.7 & 1018 & & 2.9 \\
\hline 35 & $=1$ & 15 & 8.3 & 103.7 & & 1.0 \\
\hline 40 & 1 & 15 & 7.1 & 97.9 & & 6.8 \\
\hline 15 & 7 & 40 & 1.5 & 114.0 & 9.3 & \\
\hline
\end{tabular}

Zero of velocity curve, $6^{\circ}$. Thermal constant of curve, $104.7^{\circ}$.

Table 24 shows an apparent acceleration of development under outdoor conditions over that observed at constant temperature of the same value. This acceleration is slight in extent, at its greatest being little more than the variation encountered in different lots at constant temperatures. The data, however, represent several groups of insects, not less than 1,000 individuals in each test, and the nature of the variation was consistent, never being in the plus direction but always in the minus, not only in the average shown in the table but also among the individual lots. It seems safe to conclude that there is some acceleration which may be attributed to outdoor conditions as compared with incubator conditions.

Lehenbauer (1914) in his work with maize seedlings found that the rate of growth showed a decrease when the temperature was kept at a constant value. This is further evidence for the conclusion that constant temperatures may present an abnormal and, therefore, detrimental, environment for some organisms. 
TABLE 26.-Development of blowfly larvae at temperatures changed at six or twelve hour intervals; the temperature for each six-hour period being indicated.

\begin{tabular}{|c|c|c|c|c|c|c|c|}
\hline \multicolumn{4}{|c|}{ Temperatures } & \multirow{2}{*}{ Mean } & \multirow{2}{*}{$\begin{array}{l}\text { Time } \\
\text { Days }\end{array}$} & \multirow{2}{*}{$\begin{array}{l}\text { Acc. } \\
\text { Temp. }\end{array}$} & \multirow{2}{*}{$\begin{array}{l}\text { Variation from } \\
\text { Thermal Const. }\end{array}$} \\
\hline 1 & 2 & 3 & 4 & & & & \\
\hline $20^{\circ}$ & $25^{\circ}$ & $20^{\circ}$ & $15^{\circ}$ & $20^{\circ}$ & 66 & $92.4^{\circ}$ & $-123^{\circ}$ \\
\hline 10 & 20 & 30 & 20 & 20 & 68 & 95.2 & -9.5 \\
\hline 10 & 30 & 30 & 10 & 20 & 7.0 & 980 & -6.7 \\
\hline 5 & 25 & 25 & 25 & 20 & 6.8 & 95.2 & -9.5 \\
\hline 15 & 25 & 25 & 15 & 20 & 69 & 96.6 & -8.1 \\
\hline 15 & 25 & 35 & 25 & 25 & 5.35 & 101.6 & -3.1 \\
\hline 20 & 25 & 30 & 25 & 25 & 55 & 104.5 & -.2 \\
\hline
\end{tabular}

Zero of velocity curve, $6^{\circ}$. Thermal constant of curve, 104.7 , S. D., $6.9^{\circ}$.

TABLE 27.-Development of blowfly pupae at temperatures changed at six or twelve hour intervals; the temperature for each six-hour period being indicated.

\begin{tabular}{|c|c|c|c|c|c|c|c|}
\hline \multicolumn{4}{|c|}{ Temperatures } & \multirow{2}{*}{ Mean } & \multirow{2}{*}{$\begin{array}{l}\text { Time } \\
\text { Days }\end{array}$} & \multirow{2}{*}{$\begin{array}{l}\text { Accum. } \\
\text { Temp. }\end{array}$} & \multirow{2}{*}{$\begin{array}{l}\text { Variation from } \\
\text { Thermal Const. }\end{array}$} \\
\hline 1 & 2 & 3 & 4 & & & & \\
\hline $20^{\circ}$ & $25^{\circ}$ & $20^{\circ}$ & $15^{\circ}$ & $20^{\circ}$ & 9 & $117^{\circ}$ & $-13.1^{\circ}$ \\
\hline 10 & 20 & 30 & 20 & 20 & 8.9 & 115.6 & -14.5 \\
\hline 10 & 30 & 30 & 10 & 20 & 9.2 & 119.6 & -10.5 \\
\hline 15 & 25 & 25 & 15 & 20 & 9 & 117 & -13.1 \\
\hline 10 & 15 & 40 & 15 & 20 & 9.5 & 123.5 & -6.6 \\
\hline 15 & 25 & 35 & 25 & 20 & 6.8 & 122.4 & -7.7 \\
\hline 20 & 25 & 30 & 25 & 25 & 7 & 126 & -4.1 \\
\hline 10 & 20 & 10 & 20 & 15 & 15.8 & 126.4 & -3.7 \\
\hline
\end{tabular}

Zero of the velocity curve, $7^{\circ}$. Thermal constant of curve, $130.1^{\circ}, \mathrm{S} . \mathrm{D}$., $5.7^{\circ}$.

Tables 26 and 27 show a similar acceleration when temperature variations were provided within the constant temperature incubators by moving the insects from one to another. Here again the variation is not usually greater than the deviation from the constant temperatures but it is without exception a minus variation. The significant fact about the variation in these tables is sinilar to that observed under the outdoor conditions; that is, that the variation from the calculated thermal constant is always a minus variation.

Table 25 gives the results from a series where the temperature was changed only once during the course of development, being kept for a period at one constant temperature and after one or more days changed to another constant and allowed to remain at that point until development was completed.

The variations from the thermal constant of the velocity curve are slight, within the standard deviation and occur in both the plus direction and the minus direction. They indicate no stimulating, or other, effect produced by a single change of temperature during 
the developmental period so it seems that variation, to cause acceleration, must be daily in its occurrence or at least must occur with relative frequency.

Why a stimulation should occur from variation in temperature is not clear. A speculative reason might be that the protoplasm of these organisms is adapted to variable conditions and that variable tempertures constitute a normal environment while constant temperatures are abnormal and so retard development. This would interpret the difference in rate as a retardation due to constant temperatures rather than as an effect of the stimulation from variation of the temperature.

It is reasonable to conclude that the acceleration resulting from outdoor conditions as compared with constant temperatures within the incubators is due to the fact of variation rather than to any other change in the conditions and it follows that computations from field observations will have to consider a thermal constant somewhat lower than one which may be established from constant temperatures within the laboratory. The results here presented do not show sufficient uniformity to suggest any method for the correction of constant-temperature constants so that they may be applied to field temperatures.

The foregoing statements should not be taken to indicate that there are no field conditions other than variations in temperature which may influence the rate of development.

\section{Carbon Dioxide Production at Different Temperatures}

The theory of the thermal constant suggests the idea that development may represent a constant quantity of metabolism. If this is true, it would be natural to expect that other indices of the quantity of metabolism should show some approach to a constant. Krogh (1914 and 1916) has reported some work which indicates a relatively constant total carbon dioxide production during a given developmental period. The work here reported was undertaken therefore, to throw additional light upon the problem of the constant quantitative relations of development.

\section{CARBON DIOXIDE ESTIMATION}

Special apparatus used in the estimation of the carbon dioxide production of insects was simple. At first it was thought necessary to make the absorption of the carbon dioxide continuous. It was later found that no apparent difference in results was caused if the carbon dioxide was removed from the breeding tubes only once daily and this practice was adopted. For this work tubes about two 
inches by six inches were used as containers. These were open at both ends and were stoppered with rubber stoppers perforated for drainage tubes. The drainage tubes were connected through the walls of the incubators with the container for the absorption of the gas. Once each day a stream of carbon dioxide-free air was passed through the tubes and the carbon dioxide contained in the tubes carried with the air through an absorbing solution containing sodium hydroxide of standard strength to an indicator solution of sodium phosphate and phenol-sulphone-phthalein. The amount of carbon dioxide was calculated, at the end of the test, by a comparison of the indicator solution with standard solutions of known acidity. These were made by adding known quantities of hydrochloric acid to the standard indicator solution. Color comparison was relied upon to determine the degree of change.

While this method cannot pretend to absolute accuracy it is probably as reliable for the small quantities of carbon dioxide involved as any other available method. It is probable that the errors in the different tests were of the same order of magnitude and not great enough to invalidate such tentative conclusions as have been drawn from this phase of the work.

Weighed lots each containing 40 grams of pupae of the blowfly, about 500 individuals being included in each lot, were used in this study. Those lots of insects kept at the lower temperatures failed to complete their development by the end of 62 days and so were discarded, examination subsequently showing that they were nearly all dead. Table 28 summarizes the results.

\section{TABLE 28.-Calliphora vomitoria pupae. Zero of velocity curve $7^{\circ}$ to $7.3^{\circ}$.}

\begin{tabular}{c|c|c|c|c}
\hline \hline Tempt. & No. of Lots & Total Grams CO & Time in Days I Average per Day \\
\hline $30^{\circ}$ & 16 & 25946 & 6 & 4324 \\
20 & 15 & 27022 & 97 & 2785 \\
10 & 12 & 28910 & 49 & 0590 \\
8 & 4 & 13224 & 62 & 0213 Discontinued \\
7 & 4 & 3869 & 62 & 0062 Discontinued \\
5 & 2 & 1440 & 62 & 0008 Discontinued \\
\hline
\end{tabular}

The three lots at the lower temperatures, since they were discontinued before development was complete, and since many pupae were dead before the experiments were diseontinued show merely that metabolism was going on at these temperatures and indicate that it was more rapid at $8^{\circ}$ than it was at $7^{\circ}$ or $5^{\circ}$. Since it was not known how many of the pupae were dead in each lot at different stages of the experiment, the latter conclusion is not established 
beyond doubt as it is entirely possible that large numbers of survivors at the higher of these temperatures explain the differences in quantity.

The carbon dioxide production at the three higher temperatures is shown to be nearly the same in amount. The percentage of emergence was approximately the same in the three lots or series. There is shown, in the results, a slightly greater production total at low temperatures than at high. This may easily be due to experimental error since neither the method nor its application pretends to absolute accuracy. There is, however, another explanation which, while not established, may be suggested. The results show that carbon dioxide was produced at temperatures below those at which development has been apparent in any of the other experimental work. This indicates a certain amount of respiration concerned not with development but with the mere maintenance of life. This may be ascribed to basal metabolism. It is reasonable to assume that this basal metabolism continues within the range of developmental temperatures. It is also possible to assume that it becomes progressively greater with increased temperatures, although there is no evidence offered here which will completely support that assumption.

If we assume the basal metabolism to persist through the range of temperatures where development is possible, regardless of whether it decreases or increases, we may find a possible explanation of at least a part of the discrepancy noted in the total carbon dioxide production at the different temperatures. The observed amounts may represent a constant total developmental metabolism plus a more or less constant daily basal metabolism repeated for the number of days which were required for development. Assuming the production at $7^{\circ}, 0.0062$ grams daily, to represent basal metabolism for any temperature, we have then to subtract 0.0062 times 6 days from the $30^{\circ}$ total; 0.0062 times 9.7 days from the $20^{\circ}$ total and 0.0062 times 49 days from the $10^{\circ}$ total. If this be done we have for the corrected total representing development only; at $30^{\circ}, 2.5574$ grams ; at $20^{\circ}, 2.6421$ grams ; and at $10^{\circ}, 2.5872$ grams, which brings the value of the totals as near to a constant as could be expected from the method used.

Whether this treatment of the data is justifiable or not must be proven by subsequent investigation. In any event the carbon dioxide production totals at the three temperatures are decidedly of the same order of magnitude and offer no difficulty in the way 
of the acceptance of the thermal constant as indicating that the processes of development represent a definite total of metabolic activity which may require a definite amount of heat for its accomplishment.

Attempts were made, early in the study of the carbon dioxide production, to make comparisons on the basis of daily production at different temperatures. This was found to be impossible because the production, at any one temperature, is not the same day after day but varies widely according to the internal condition of the pupae. This fact has been well established by other investigators; Tangl (1909), Krogh (1914 and 1916), Sosnowski (1902) and Weinland (1906) all mention it. It is necessary, therefore, in comparative work, to consider the entire developmental period or to compare a day at one temperature with the period of time at a second temperature which has been experimentally demonstrated to be equivalent to a day at the first temperature, basing comparisons on the amount of development accomplished during the period. It is necessary also that the same portions of the developmental period be compared with each other; that is, it would not be correct to compare a period representing the first one-fourth of the total period with another representing the second one-fourth.

While the results from the comparative daily measurements do not bear directly on the problem they may be included here since they serve to illustrate the point just made.

TABLE 29.-Carbon dioxide production of pupae of C. vomitoria by days. Average in grams $\mathrm{CO}$. for three lots of $\mathbf{4 0}$ grams each at each temperature.

\begin{tabular}{|c|c|c|}
\hline Day & Production at $30^{\circ}$ & Production at $20^{\circ}$ \\
\hline Ist & .5062 & 2043 \\
\hline $2 \mathrm{nd}$ & .4694 & 2969 \\
\hline 3rd & 2045 & .3756 \\
\hline 4th & 1920 & 3406 \\
\hline 5 th & 4642 & 3025 \\
\hline 6 th & .5501 & 2962 \\
\hline 7 th & & 2128 \\
\hline 8 th & & 1440 \\
\hline 9 th & & .1606 \\
\hline 10th & & 1682 \\
\hline Totals & 23866 & 2.5216 \\
\hline
\end{tabular}

The totals given in Table 29 do not represent quite the entire production as some of the pupae were discarded after the majority had emerged in each of the lots. 


\section{Moisture}

The estimation of the effects which variations in moisture either in the atmosphere or in the soil surrounding the organisms or in their food has been no part of the purpose of this paper. That variation in atmospheric and other moisture may affect the rate of development or inhibit it entirely has been abundantly demonstrated.

A small series of moisture tests was made, not for the purpose of studying moisture relations in general-but merely to determine whether such variations in moisture as might occur in the experimental material used in temperature work could be expected to have any material importance in the interpretation of results. These constituted a comparison of high atmospheric moisture (80 per cent relative humidity or higher), with low moisture (30 per cent relative humidity or lower), at several temperatures and with several insects. The results are given in Table 30.

TABLE 30.Development of insects under variable moisture conditions.

\begin{tabular}{|c|c|c|c|c|c|c|}
\hline \multirow[b]{2}{*}{ Insect } & \multirow[b]{2}{*}{ Stage } & \multirow[b]{2}{*}{ Temp. } & \multicolumn{2}{|c|}{ High Moisture $(80 \%)$} & \multicolumn{2}{|c|}{ Low Moisture $(30 \%)$} \\
\hline & & & $\begin{array}{c}\text { Time in } \\
\text { Days }\end{array}$ & $\begin{array}{l}\text { Per Cent } \\
\text { Emerged }\end{array}$ & $\begin{array}{c}\text { Time in } \\
\text { Days }\end{array}$ & $\begin{array}{l}\text { Per Cent } \\
\text { Emerged }\end{array}$ \\
\hline C. vomitoria & pupae & $30^{\circ}$ & 6 & 81 & 5.85 & 23 \\
\hline C. vomitoria & pupae & 20 & 92 & 88 & 9.35 & 46 \\
\hline C. vomitoria & pupae & 15 & 16.8 & 78 & 19 & 39 \\
\hline L. Caesar & pupae & 30 & 5 & 64 & 5.25 & 47 \\
\hline L. Caesar & pupae & 20 & 9.1 & 85 & 9.7 & 67 \\
\hline L. Caesar & pupae & 15 & 14.5 & 74 & 14.75 & 62 \\
\hline S. Carnaria (?) & pupae & 30 & 5.8 & 86 & 6. & 69 \\
\hline S. Carnaria (?) & pupae & 20 & 9.1 & 82 & 9. & 71 \\
\hline S. Carnaria (?) & pupae & 15 & 16.2 & 70 & 164 & 64 \\
\hline L. Caesar & larvae & 30 & 4. 6 & 67 & 4. 7 & 60 \\
\hline L. Caesar & larvae & 20 & 7.5 & 85 & 7.4 & 72 \\
\hline L. Caesar & larvae & 15 & 12.1 & 78 & 12.3 & 71 \\
\hline
\end{tabular}

Moisture conditions as extreme as those represented in Table 30 did not occur with any of the fly material studied. Variations indicated in the table are slight, no greater than may be found in the individual lots comprising any one of the averages given in the constant temperature tables. There is to be noted a decrease in percentage of emergence in the dry lots which is probably of significance. Likewise, in the dry lots at the higher temperature, and to a less extent at all temperatures, a reduction in the size of the emerging individuals was frequent as was also an increase in the percentage of imperfect flies.

These results merely indicate that the variations in atmospheric moisture had little effect under the conditions of these ex- 
periments. Moisture conditions of the food of the larvae and of the soil surrounding the pupae were kept as near to the standard as possible, but the extremely rapid drying under the dry conditions caused some variation in the superficial layer of sand.

Elimination of consideration of moisture variations as an important factor seems to have been justified.

\section{Sources of Error}

Attempt has been made to reduce experimental error to a minimum in this work but it is still probable that small errors of various natures contribute to the variability of the results. Temperature has been controlled to within four-ninths of a degree $\mathrm{C}$. plus or minus. It is still possible that the variation in some of the lots was consistently in one direction while in other lots it was in the reverse direction. Where the period was long, the error thus accumulated, even though it amounted to only one-fourth degree, would be great enough to account for nearly any of the variations which have been recorded. In a similar manner, a discrepancy of a fraction of a degree in the calibration of a thermometer or in the reading of it, would accumulate an error proportional to the length of the period involved. In the first instance the error would be always in one direction; in the second, if the reading were done by one person, the error would be likely to be in one direction.

Still another possible source of error based upon temperature records is the variation in temperature within the incubator. Every effort was made to have this temperature uniform within what were considered satisfactory limits and the records show that this was done. At the same time the possibility of a variation of not to exceed one-half degree which might well have been rather consistently in one direction, must be accepted and would further contribute to the general variability of the results.

The time factor introduces another source of error. Observations, as has been stated, were made at six hour intervals where the developmental period was expected to be less than twenty days and usually at similar intervals regardless of the length of the developmental period. Time of emergence or pupation was recorded as at the time of observation or as having taken place in the middle of the preceding six hour period, experience with the appearance of emerging or pupating individuals enabling one to tell with considerable certainty which record would most nearly approximate the actual conditions. Nevertheless, a possible maximum error up to six per cent must be admitted. This error will tend to have a greater value where the developmental period is short than where it is 
long and will probably be more frequently in the plus direction than in the minus.

Variation in the material used would include possible seasonal variation, the work having been done at all seasons of the year when material was available; year to year variation, the summaries including, in the fly material, insects collected during at least five different years and the individual variation which occurs among the individuals of any lot originating from a single mass of eggs or from masses collected at any one time and place. Locality variations are eliminated because all material used was collected in one restricted locality, probably all within one hundred yards of one spot.

It is not to be supposed that the observational errors, or possibility of observational errors were disregarded; every precaution was taken to exclude them and it is certain that they were not more important, at the worst, than has been suggested above. In order that these unavoidable errors might be equalized, large numbers of individuals were used wherever that was possible so that small variations would tend to cancel each other and the general average represent as nearly as possible the true conditions.

\section{Discussion of the Data}

\section{CONSTANT TEMPERATURE SERIES}

In a previous paper, (Sanderson and Peairs, 1914), the writer concluded, independent of previous work, that the developmental curve was an hyperbola and its reciprocal, the curve showing relative velocity of development, was, in its ideal form, a straight line. From these conclusions it was assumed that the threshold of development (developmental zero was the name applied at that time) should, theoretically, be the zero of the velocity curve or the point at which that curve intersected the temperature axis. The data in this study have been collected largely for the purpose of determining whether the earlier conclusions were justified. An inspection of the graphs showing the velocity curves for the material of this study will show that the points on these curves at least approach the straight line condition. To show the closeness of this approach the summary in Table 31, based on the data from the fly material only, has been prepared. This shows the percentage of departure of points of all the curves, from the calculated straight line. Departures on the curves based on data from the other insects are somewhat greater but there is a similar distribution of plus and minus values. 
TABLE 31.-Departure, expressed in percents, or points on the velocity curves, from the theoretical straight line curve.*

\begin{tabular}{|c|c|c|c|c|c|c|c|c|c|c|}
\hline \multirow{2}{*}{ Temp. } & \multirow{2}{*}{$\begin{array}{l}\text { Mean or } \\
\text { First Em. }\end{array}$} & \multicolumn{8}{|c|}{ Table's Included (Numbers) } & \multirow{2}{*}{$\begin{array}{l}\text { Average } \\
\text { in } \\
\text { Percent }\end{array}$} \\
\hline & & 1 & 2 & 3 & 4 & 5 & 6 & 7 & 8 & \\
\hline $35^{\circ}$ & $\begin{array}{l}\text { Mean } \\
\text { First }\end{array}$ & $\begin{array}{r}-1.6 \\
-\quad 5\end{array}$ & $\begin{array}{c}-1.5 \\
5 .\end{array}$ & $\begin{array}{ll}-6 & \\
-1 & 1\end{array} \mid$ & $\begin{array}{r}-1.5 \\
15\end{array}$ & $\begin{array}{c}-1.6 \\
1 .\end{array}$ & $-\begin{array}{r}.6 \\
1.8\end{array}$ & $\mid \begin{array}{rr}-2 & 2 \\
1 & 6\end{array}$ & ${ }^{-1}$ & $\begin{array}{ll}2 & 12 \\
1 & 6\end{array}$ \\
\hline 30 & & $\begin{array}{r}-2.7 \\
-\quad .8\end{array}$ & $\begin{array}{r}-3 . \\
12\end{array}$ & $\begin{array}{r}9 \\
-\quad 1\end{array}$ & $\begin{array}{rr}28 \\
& 8\end{array}$ & $\begin{array}{r}2.5 \\
.2\end{array}$ & $\begin{array}{r}.2 \\
1.2\end{array}$ & $\begin{array}{r}1.2 \\
.7\end{array}$ & $\begin{array}{l}4 \\
3 .\end{array}$ & $\begin{array}{ll}1 & 7 \\
1 & 0\end{array}$ \\
\hline 25 & $\begin{array}{l}\text { Mean } \\
\text { First }\end{array}$ & -7.2 & $\begin{array}{l}-2.5 \\
-1 .\end{array}$ & -2.2 & -3.7 & $\begin{array}{l}2.6 \\
1.3\end{array}$ & -6. & $\begin{array}{l}-2.2 \\
2 .\end{array}$ & -8. & $\begin{array}{ll}3 & 9 \\
1.15\end{array}$ \\
\hline 20 & $\begin{array}{l}\text { Mean } \\
\text { First }\end{array}$ & -3.9 & $\begin{array}{l}2.0 \\
1.7\end{array}$ & $\begin{array}{r}-2.5 \\
-\quad 8\end{array}$ & -5.6 & $\left|\begin{array}{r}0.0 \\
-2.8\end{array}\right|$ & -5 & -4.5 & 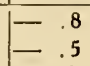 & $\begin{array}{l}28 \\
1.6\end{array}$ \\
\hline 15 & $\begin{array}{c}\text { Mean } \\
\text { First }\end{array}$ & $\begin{array}{l}3.0 \\
2.0\end{array}$ & $\begin{array}{r}02 \\
-3.0\end{array}$ & $\begin{array}{l}0.6 \\
2.2\end{array}$ & -4.6 & $-\begin{array}{r}.8 \\
-1.6\end{array}$ & $\begin{array}{r}10 \\
2\end{array}$ & $\begin{array}{r}7 . \\
-\quad .6\end{array}$ & $\begin{array}{l}7 . \\
1.2\end{array}$ & $\begin{array}{l}4.05 \\
1.65\end{array}$ \\
\hline 10 & $\begin{array}{l}\text { Mean } \\
\text { First }\end{array}$ & $\begin{array}{l}8 . \\
4 .\end{array}$ & $\begin{array}{r}10 \\
4\end{array}$ & $\begin{array}{l}4.5 \\
2 .\end{array}$ & $\begin{array}{l}8 . \\
4 .\end{array}$ & -4 & $\begin{array}{l}-4 \\
-2\end{array}$ & $\begin{array}{r}10 . \\
6 .\end{array}$ & $\begin{array}{l}25 . \\
11 .\end{array}$ & $\begin{array}{l}9.2 \\
5.6\end{array}$ \\
\hline 8 & $\begin{array}{l}\text { Mean } \\
\text { First }\end{array}$ & 11.8 & $\begin{array}{r}25 . \\
8\end{array}$ & $\begin{array}{l}7.5 \\
3.5\end{array}$ & $\begin{array}{r}24 . \\
-4 .\end{array}$ & $\begin{array}{l}5 . \\
8\end{array}$ & $\begin{array}{r}13 \\
4\end{array}$ & & & $\begin{array}{l}14 . \\
8.1\end{array}$ \\
\hline $\begin{array}{c}\text { Aver, for } \\
\text { Table }\end{array}$ & $\begin{array}{l}\text { Mean } \\
\text { First }\end{array}$ & $\begin{array}{l}5.3 \\
2.3\end{array}$ & $\begin{array}{ll}6 & 3 \\
3 & 4\end{array}$ & $\begin{array}{l}3.4 \\
1.7\end{array}$ & $\begin{array}{l}6.9 \\
1.9\end{array}$ & $\begin{array}{l}2.36 \\
3.3\end{array}$ & $\begin{array}{l}5.5 \\
2.3\end{array}$ & $\begin{array}{l}4.6 \\
1.6\end{array}$ & $\begin{array}{l}7.6 \\
2.8\end{array}$ & $\begin{array}{l}5.25 \\
2.4\end{array}$ \\
\hline
\end{tabular}

*Tested by Harris' method for determining goodness of fit, the formula being

$$
X^{2}=S \frac{(o-c)^{2}}{c}
$$

where $\mathrm{o}$ is the observed value for the "constant" at any point, while $\mathrm{c}$ is the calculated value for the same point, the values for $\mathrm{X}^{2}$ being converted into $\bar{a}$ expression of the chances favoring the straight line interpretation of the data, the values for the mean and for the minimum time emergence data from tables 1 to 8 , give the following:

$\begin{array}{lrr}1 & 38-1 & 99-1 \\ 2 & 53-1 & 99-1 \\ 3 & 96-1 & 99-1 \\ 4 & 99-1 & 99-1 \\ 5 & 95-1 & 98-1 \\ 6 & 70-1 & 98-1 \\ 7 & 96-1 & 99-1 \\ 8 & 10-1 & 93-1\end{array}$

In practically all cases these values indicate a certainty that the straight line is the proper interpretation for the data. It is to be noted that the values indicate clearly that the use of the minimum emergence data is preferable.

Table 31 shows that there is a difference in the degree of error indicated in the mean curves and in the first emergence curves, the latter being consistently lower. There is also a difference in the distribution of the errors. The mean temperature summary shows that variations at high temperatures are all in the minus direction while those at the lower temperatures, $8^{\circ}$ and $10^{\circ}$, are nearly all in the plus direction. The reason for the minus variation at $35^{\circ}$ is probably that the low temperature plus variations are rather large and that these have shifted the position of the curve so that the true nature of the variations at $35^{\circ}$ may have been obscured.

In the summary of first emergence data, on the other hand, the low temperature variations are seen to be much smaller so that they have had less effect in changing the position of the curve relative to the high temperature points; these latter now show a decided tendency to vary in the plus direction and this probably indicates 
the beginning of the high temperature retardation effect. This high temperature retardation has not been emphasized in the data presented but has been generally noted. At points ranging from about $35^{\circ}$, more or less, the development is slowed down. Rarely is complete development possible at $40^{\circ}$. This fact has been brought out by other investigators (Krogh, 1914) and seems to be a general phenomenon.

No evidence which would tend to suggest an experimental explanation of this behavior has been offered. Probably the limits of what is termed normal development occur about this point on the temperature scale. The lethal effect of high temperatures, which may have to do with changes in the chemical condition of the protoplasm, possibly due to colloidal changes, may show here the first evidences of their action. For a limited range of temperature, development although retarded, is still complete. Somewhat higher temperatures still permit partial development but death intervenes before the process is complete. Such lethal effects may actually be present at lower temperatures but be too slight in extent to be noticeable.

Departures from the mean, indicated in Table 31, for median temperatures, particularly those based upon first emergences, are slight, usually well within the limits of observational error, and show a fairly even distribution between plus and minus aberrations.

The summary indicates that the averages based upon time of first emergence are probably a better expression of the temperature effect than are those based upon mean emergence.

The extent and nature of the variations from the constant indicated in the summary are the strongest evidence presented that the straight line is the true shape of the curve of velocity of development, at median temperatures. Near the lower end of the curves, a rather consistent variation in the plus direction, indicating retardation of development and a greater time requirement, is found. Even here, the daily value of such variations is extremely small; their considerable effect in the total thermal increment is due to the fact that they are repeated daily for a period of many days.

Explanation of this retardation may readily be found in the developmental conditions of both larvae and pupae. In the case of larvae, the necessity for changing the food material when the developmental period lasted much longer than it probably ever does under normal conditions outdoors, causes some delay incident to 
the transfer of larvae from the old food to the new and to the fact that they do not begin feeding immediately upon new food. This might easily account for such retardation as has occurred with larvae.

Pupae, when their development requires a long period, regardless of the care with which temperature and moisture conditions are maintained, show a change in the texture of the outer covering or puparium. This may be likened to a ripening process or it may better be called merely aging. These puparia are tougher in their texture than the fresher ones which undoubtedly delays the emergence of at least the weaker individuals in a group and certainly prevents entirely the emergence of many of them, thus accounting for a part of the mortality always observed in low-temperature lots.

It is to be noted that these possible factors will both cause retardation in development; that is, variation in the plus direction, which is in accord with the observations. It is likewise probable that they will operate less noticeably with the more vigorous individuals in any lot so that the time of first emergence might be expected to be less influenced than the mean. This agrees with results also, the variations from the straight line being less marked when the curve is based upon first emergence than when it is based upon the mean emergence time.

The foregoing discussion applies only to evidence presented in this paper. So far as that evidence alone is concerned there is no reason to believe that the straight line interpretation might not apply to all parts of the curve below certain upper limits as has been previously pointed out.

A considerable amount of data from other sources indicates that the developmental process at low temperatures within a few degrees of the zero of the velocity curve, cannot be expressed by the continuation of the straight line but that there is a distinct aberration in the direction which indicates acceleration.

Shelford (1917) has called particular attention to this point and the work of Krogh (1914), Reibisch (1902), Loeb and Wasteneys (1911), Loeb and Northrup (1917), Headlee (1914), and Krafka (1920), to mention only a few of those bearing on this point, as well as a more recent study by Shelford (1926), have indicated clearly this deviation in the minus direction. In some cases this deviation has been so great that development has been demonstrated at temperatures below the zero of the velocity curve. This was shown by Loeb and Northrup (1917) as pointed out by 
Krafka (1920). Shelford has found a similar condition with the codling moth while the work of Krogh suggests that the condition holds true for several animals studied by him.

In none of these instances is the variation great in degree but the fact that it has been observed by several different investigators working with diverse materials precludes any explanation on the basis of experimental error. The uniformity of the conclusions which these several investigations demonstrate, points strongly to a probability that the threshold of development is actually below the zero of the velocity curve.

Apparently the only contrary evidence is contained in this paper. Of the fact of retardation here recorded, there can be no doubt. Nor can there be doubt as to the evidence showing that there was no apparent development at the zero point even with flies kept at that temperature for periods extending as long as twenty-three months. The improbability of a fundamental difference, at this point, of the temperature effect on different organisms, leads to the search for an explanation which may reconcile the conflicting data.

It has already been pointed out that the retardation of developmental rate as compared with that to be expected from the straight line curve, might be explained by the operation of outside factors, variation in food supply of larvae, and change in the texture of the puparium, not connected directly with temperature. It is certainly possible that these same factors might account for the somewhat greater variation from the condition of the curve representing a lower threshold of development.

The lack of evidence of development at the zero point has been mentioned. Slight development at temperatures one degree or onehalf degree above this zero has been recorded but this has always been less than the time at these temperatures would lead one to expect. This shows that there is a factor for retardation operating at low temperatures. It is conceivable that this retardation is so great at the next lower temperatures studied as entirely to obscure the evidence of any slight development which might take place.

It might be expected that the zero of the developmental curve would be the zero, or threshold, of development. Actually, it seems that it is not. A probable explanation is that development begins at a point somewhat lower than the zero of the curve; that it is at first very slow but gradually increases in rate of acceleration until the protoplasm reaches the condition of stability which it maintains through the normal range of development. From this point of 
stability the curve of increase in velocity runs in a straight line until it reaches the point of falling-off near the upper limit of the developmental temperatures. This condition represents a gradual increase in the value of the day degree from zero, at the threshold, to a constant value at the point of stabilization of the protoplasm.

Discussions up to this point have dealt with the applicability of the theory of the interpretation which assumes the developmental curve to be an hyperbola and its reciprocal, the straight line, to represent the rate of increase in the velocity of development. Whether this is the best explanation of the facts must be determined by the consideration of other explanations which have been offered. Most prominent among these has been the law of van't Hoff.

The law of van't Hoff (1884) states that the velocity of a chemical reaction increases with the temperature and that the coefficient expressing the rate of increase is usually between two and three for each ten degree rise in temperature. Actually, over any considerable temperature interval, the coefficients show a much greater variation than this and it seems to have been no part of the assumption in the original formulation that the coefficient was, for any ten degree interval, the same as for any other ten degree interval.

Observations in the field of biology showed that the actual coefficient of increase in growth or rate of development was frequently between two and three and this led to the use of the van't Hoff formula as a convenient means for describing the rate of increase in terms which might refer the origin of the increased velocity to chemico-physical causes.

An examination of the coefficients for a chemical reaction and a comparison of these with similar coefficients for a biological reaction, will show that they are similar in order of magnitude; that they decrease with each successive rise in temperature; and that they show, graphically, the same relation to the straight line as do the developmental data. If, as is apparent, the van't Hoff coeffcients are not constant they can have no value in other than a descriptive sense, correlating the biological process with the chemicophysical, unless a method for the calculation of the one from the other can be devised. The straight line interpretation of the velocity curve seems to furnish this.

It appears, therefore, that the van't Hoff coefficients as used in the description of the rate of chemical reactions, apply in a sense to biological processes, but that they vary in their magnitude and may best be calculated from the straight line index. 
Krogh (1916) reaches conclusions essentially similar to the foregoing. Kanitz (1915) brings out the similarity between the biological coefficients and those for chemical reactions.

Arrhenius' modification of van't Hoff's formula has also been suggested as a possible basis for the explanation of the biological problem. It is open to the same objection as the van't Hoff formula in that the coefficients derived from its application are not ordinarily constant. As applied to data in this paper, the Arrhenius' coefficients plot graphically on an approach to the straight line index (van't Hoff coefficients plot graphically on the hyperbola).

Crozier (1924) has found that for certain reactions of organisms to stimuli as influenced by temperature changes, the Arrhenius' coefficient (termed "temperature characteristic" by Crozier) is a constant for a certain part of the temperature range, but that at a temperature of usually about $16^{\circ}$, the constant changes and another holds from that point downward. Crozier interprets this by assuming that the reaction is first based upon some one single chemical reaction of a simple nature; that at the point where the second coefficient enters a different reaction begins to take place. No parallel between these phenomena observed by Crozier and the developmental data is apparent. It is entirely probable that the vital reactions in development are affected by so many different chemical processes that we are not yet ready to attempt to isolate the effects of the individual ones. Krogh found the formula of Arrhenius to be no more satisfactory than that of van't Hoff in the explanation of data which was essentially similar to that contained herein.

Of both these formulae it may be said that they do not conflict with any of the conclusions involving the straight line interpretation of the velocity curve but that neither is as useful for study of biological data as is the study of such data in relation to the straight line.

The formula of van't Hoff is as follows:

$$
Q 10=\frac{K_{2}}{K_{1}}\left(\frac{10}{t_{2}-t_{1}}\right)
$$

Q10 being the usual designation of the van't Hoff coefficient; $K_{1}$ being the time at the first temperature; $t_{1}$ and $K_{2}$ the time at the second temperature.

Arrhenius' formula is usually given as:

$$
K_{2}=K_{1} \cdot e \frac{q}{r} \cdot \frac{T_{2}-T_{1}}{T_{2} \cdot T_{1}}
$$


In whis formula $K$ designates the time factors at the first temperature and at the second; $T$ refers to absolute temperature and " $r$ " is a constant which is approximately equal to two and is usually applied as that number; " $q$ " is then the constant for the formula or the "characteristic" according to the usage of Crozier.

For biological reactions it might be better to use effective temperatures rather than absolute temperatures since, according to the evidence, temperatures below the threshold have no influence upon development. Figure 18 shows the Arrhenius' coefficients based upon the minimum emergence data from Table 4 , in their relation to the straight line. Figure 19 shows that van't Hoff coefficients from the same data and their relation to the curve.

Other suggested explanations of the velocity curve are somewhat indefinite. That the curve, near its lower limits, may be an exponential one, as has been suggested, now seems probable. That the straight line curve may be, in fact, not one curve but two, one representing development above a given point and another development below, or one representing the action of one set of forces and the other the operation of a different set of forces, the two being assumed to overlap and the straight line being the component of the two sets of forces, is within the bounds of speculation. Since there is no definite evidence upon which to base a discussion of this possibility no use can yet be made of it.

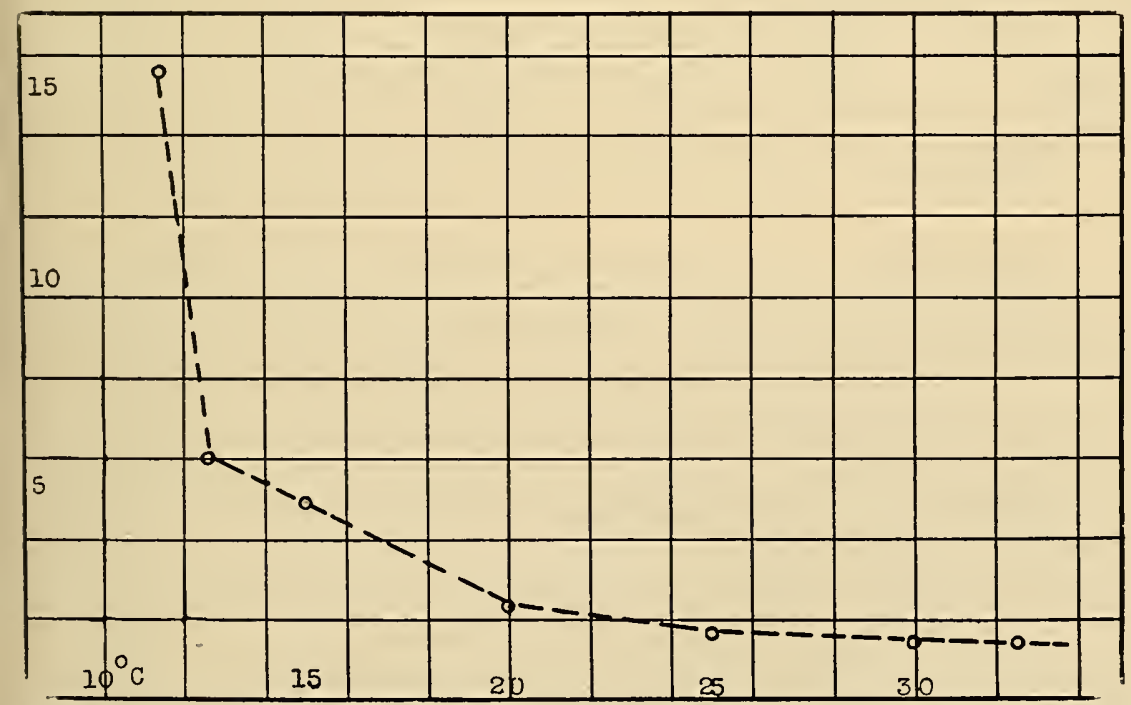

Fig. 18.-Arrhenius' coefficient based upon data in Table 4. (Effective temperatures.) 


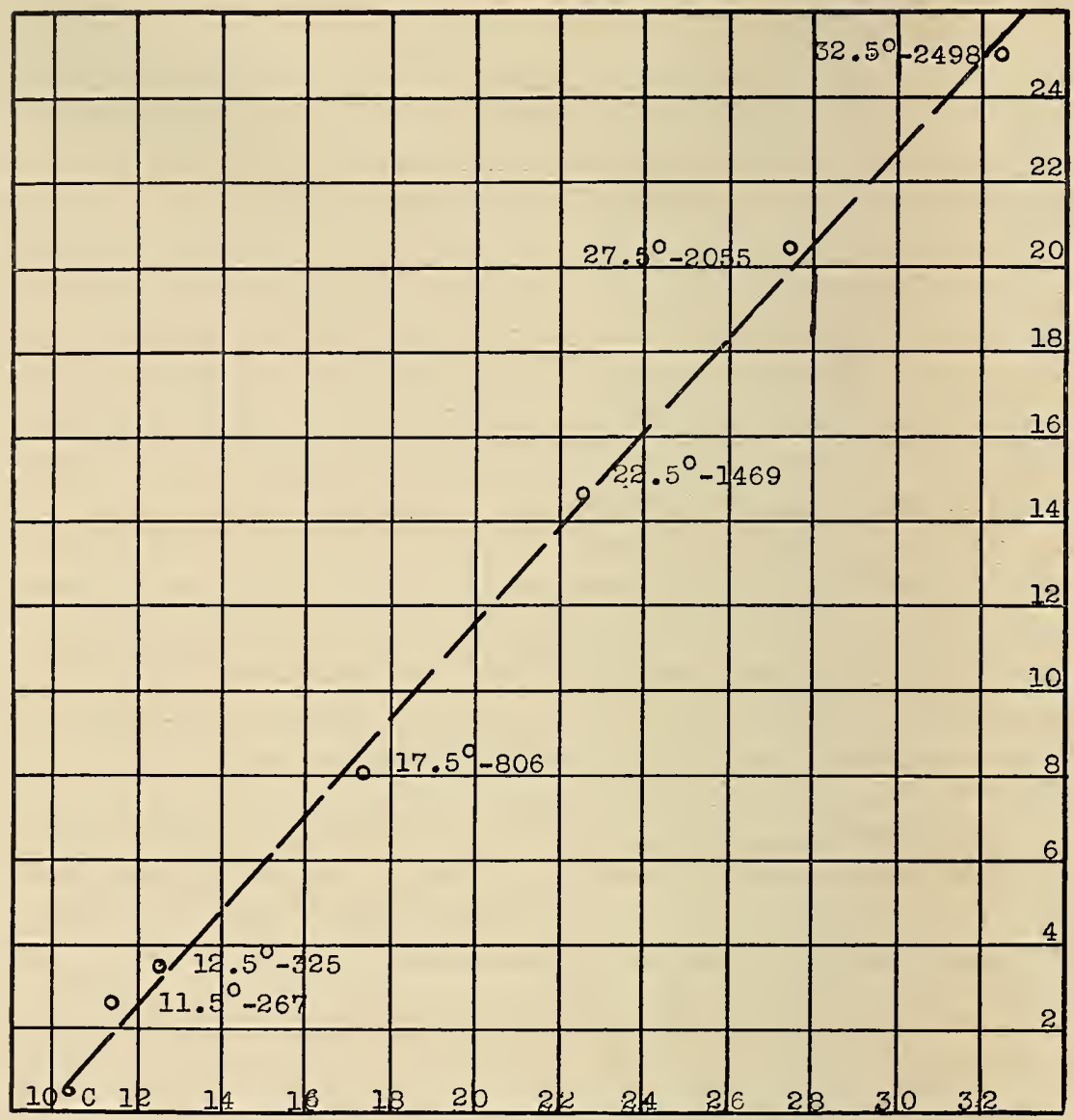

Fig 19.-Van't Hoff coefficients from data in Table 5. Based on minimum emergence records.

Still another possibility is that there is no definite law governing developmental processes. This is entirely contrary to the modern trend of interpretations of vital phenomena and absolutely untenable if we accept the principle of the essential unity of biological processes with those of chemistry and physics. Barry (1904) has pointed out that there is nothing more exact than the laws of thermodynamics and also the probability that these laws apply in biology. The whole trend of investigational results establishes this. If the data now available do not fit exactly any physical explanation it is entirely probable that the correct explanation has not been suggested or that the data are not. sufficient in quantity or in accuracy. 


\section{SUMMARY}

1.- It has been shown that the rate of development of certain insects is directly affected by the degree of temperature and that, for the greater part of the range of temperatures permitting development, this relation is best expressed by a curve for the velocity of development which takes the form of a straight line.

2. - Data indicate that the straight line departs from its course near the ends. At the upper end the departure is in the direction which indicates a slowing up of acceleration of development. The opposite condition is observed at the lower end of the curve but here, in the light of evidence presented by other investigators, it seems probable that the increase in the rate of acceleration is due to factors other than temperature and that the actual temperature effect would be represented by a departure from the straight line in the direction which would represent decrease in the rate of acceleration of development.

3.- For all points along the straight line part of the velocity curve, a given accumulation of temperature, or thermal increment, expressed in day-degrees, will accomplish a constant effect, expressed in growth or development. And, further, that summations of temperatures, to derive the constant, may be made from the zero of the velocity curve.

4.- The point at which development ceases is near to, but somewhat lower than the zero of the velocity curve. No definite method of locating this threshold of development, is suggested.

5.-Evidence of development, in the work reported here, has been lacking from insects reared at temperatures approximating the zero of the velocity curve but other investigators, working with other material, have found evidence of development at points even below the zero of the curve.

6.-Development has been found to be accelerated by variations in the daily temperature when compared with constant temperatures of the same apparent value.

7.-Some evidence is offered to indicate that the quality of carbon dioxide produced during a developmental period is near a constant, regardless of the temperature at which development takes place, and it is suggested that the departures from the constant may represent basal metabolism not directly associated with developmental processes.

8. - Data is presented which show that temperatures undergoing a daily variation similar to the normal variation found under outdoor conditions have a greater effect upon rate of development than do constant temperatures from which the same thermal increment is to be computed.

9.-It is shown in the discussions that the law of van't Hoff and possibly Arrhenius' formula also may be applied to developmental data but that the graphic interpretation, based upon the straight line curve, furnishes a more convenient method of analysis. 


\section{BIBLIOGRAPHY}

Abee, Cleveland

1878. THERMal CONSTANT FOR LOCUST EGGS. First Report of the U. S. Ent. Comm. p. $426-432$.

1905. THE RELATION BETWEEN CLIMATE AND CROPS. U. S. Weather Bureau, Bul. 36.

Appleman, C. O.

1923. FORECASTING THE DATE AND DURATION OF THE BEST CANNING STAGE FOR SWEETCORN. Md. Agr. Expt. Sta. Bul. 254.

Arrhenius, Svante.

1889. ZEITSCHR. PHYSIK. CHEM. 4.

BachmetjeW, P.

1901. EXPERIMENTELLE ENTOMOLIGISCHE STUDIEN, I. TEMEPRATUR VERHALTNISSE BEI INSEKTEN. pp. 160. Leipzig, 1901.

1907. EXPERIMENTELLE ENTOMOLOGISCHE STUDIEN VOM PHYSIKALLISCHCHEMISCHEN STANDPUNKT AUS. Sofia.

Back, E. A., and Pemberton, C. E.

1916. THE EFFECT OF COLD-STORAGE TEMPERATURES ON PUPAE OF THE MEDITERRANEAN FRUIT-FLY. Jour. Agr. Res. 6: 251-260.

BAILEY, L. H.

1896. THE SURvival OF THE UNLIKE. New York.

1889. THE INFLUENCE OF CERTAIN CONDITIONS ON THE SPROUTING OF SEEDS. Cornell Agr. Expt. Sta. Bul. 7.

Barenne, Dusser de and Burger, G. C. E.

1924. OXYGEN CONSUMPTION AND CARBON DIOXIDE OUTPUT. Jour. Phys. $59: 17-29$.

BARRY, FREDERICK

1914. THE INFLUENCE OF TEMPERATURE UPON CHEMICAL REACTIONS. Am. Jour. Bot. 1: 203-225.

BAYLISS, W. M.

1924. PRINCIPLES OF GENERAL PHYSIOLOGY. London.

Bertrand, G., Brocq-Roussen, et Dassonville.

1919. INFLUENCE DE LA TEMPERATURE ET D'AUTRES AGENTS PHYSIQUES

SUR LE POUVOIR DE LA CHLOROPICRINe. Compt. Rent. Acad. Sci. 159: 1059-1061.

\section{Beauvais}

1837. SEIDENWURMER.-ISIS vON OKEN. p. 781 (Cited from Backmetjew.)

Bodine, JoSEPH HaLL

1921. FACTORS INFLUENCING THE WATER CONTENT AND THE RATE OF METABOLISM IN CERTAIN ORTHOPTERA.. Jour. Expt. Zool. 32: 137-164.

1925. THE EFFECT OF TEMPERATURE ON THE RATE OF EMBRYONIC DEVELOPMENT OF CERTAIN ORTHOPTERA. Jour. Exp. Zool. 42: No. 1. 
CANDOLle, A. P. De

1855. GEOGRAPHique botanique RAISONEe. Paris.

1865. DE LA GERMINATION SOUS LES DEGRES DIVERS DE TEMPERATURE CONStance. Bibl. univ. et Revue suisse. Tome 14: 243-282. Geneva.

CotTon, E. C., AND Voorhees, J. F.

1911. the Cattle tick AS Affected by Climate. Tenn. Agr. Expt. Sta. Bul. 94.

CROZIER, W. J.

1924. BIOLOGICAL OXIDATION AS A FUNCTION OF TEMPERATURE. Jour. Gen. Phys. 7: 189-216.

1924.-CRITICAL THERMAL INCREMENTS FOR THE MOVEMENT OF A DIPLOPOD. Jour. Gen. Phys. 7: 123-216.

AND STEIR, T. B.

1924. CRITICAL THERMAL INCREMENTS FOR RHYTHYMIC RESPIRATORY Movements of INSECTs. Jour. Gen. Phys. 7: 429-447.

AND FredRIGHT, $\mathrm{H}$.

1924. CRITICAL THERMAL INCREMENTS FOR MOVEMENTS OF OSCILLATORIA. Jour. Gen. Phys. $7: 143-151$.

PHOTOTROPIC CIRCUS MOVEMENTS OF LIMAX AS INFLUENCED BY TEBPERATURE. Jour. Gen. Phys. 7: 151-170.

DANNEVIG, HAROLD

1894. THE INFLUENCE OF TEMPERATURE ON THE DEVELOPMENT OF THE EGGS OF FISHES. 13th Ann. Rept. Fisheries Board of Scotland. p. 147-153.

DAVENPORT, C. B.

1897 and 1899. EXPERIMENTAL MORPHOLOGY (Parts First and Second). New York.

DEAN, G. A.

1911. HEAT AS A MEANS OF CONTROLLing Mill insects. Jour. Ec. Ent. $4: 143-158$.

EDwARnS, C. L.

1902. PHYSIOLOGICAL ZERO AND THE INDEX OF DEVELOPMENT OF THE EGG OF THE DOMESTIC FowL. Am. Jour. Physiol. 6: 351-397.

\section{EDWARDS AND COLIN}

1834. INFLUENCE DE LA TEMPERATURe SUR la Germination. Ab. Sec. Nat. 2nd. Series I.

FABRE, J. H.

Souvenirs entomologiques. Troisieme Serie.

FERE, C.

1894. NOTE SUR L'INFLUENCE DE LA TEMPERATURE SUR L'INCUBATION DE L'OEUF DE POULE. Jour. de l'Anat. et de la Physiol. 30:352365.

GLenN, P. A.

1922. CODLING moth investigations. Nat. Hist. Surv. of Ill. 14: 
GreEN, SETH

1870. TRout CULTURE, Rochester, N. Y.

HARTZELL, F. Z.

1919. COMPARISON OF METHODS FOR THE COMPUTATION OF DAILY MEAN TEMPERATURES: EFFECT OF DISCREPANCIES UPON THE INVESTIGATIONS OF CLIMATOLOGISTS AND BIOLOGISTS. N. Y. Agr. Expt. Sta. Bul. 68 (Tech).

HEADLEE, T. J.

1914. THE EFFECTS OF TEMPERATURE AND MOISTURE ON THE RATE OF INSECT METABOLISM. Jour. Econ. Ent. 7: 413-417.

1917. SOME FACTS RELATIVE TO THE INFLUENCE OF ATMOSPHERIC HUMIDITY ON INSECT METABOLISM. Jour. Econ. Ent. 10: 31-38.

HeRMS, WM. B.

1907. AN ECOLOGICAL AND EXPERIMENTAL STUDY OF SARCOPHAGIDAE. Jour. Exp. Zool. IV: 45-83.

HEARSH, A. H.

1923. THE EFFECT OF TEMPERATURE UPON THE BAR-EYED RACE OF DROSOPHILA. Jour. Biol. Chem. 59: 55-73.

HERSH, ROSELLE KARRAR

1923. THE EFFECT OF TEMPERATURE UPON THE FULL-EYED RACE OF DRosopHILA. Jour. Biol. Chem. 59: 43:55.

HeNDERSON, L. J.

1913. THE FITNESS OF THE ENVIRONMENT. New York.

HERWIG, O.

1896. UEBER DEN EINFLUSS VERSCHEIDENER TEMPERATUREN AUF DIE ENTWICKLUNG DER FROSCHEIER. Sitzungsber. Berlin. Akad. Bd. 19.

1898. UEBER DEN EINFLUSS DER TEMPERATURE AUF DIE ENTWICKLUNG voN RANA FUSCA UND R. esculenta. Arch. f. Mikr. Anat. 51: $319-381$.

HILDERBRANDT, F. MERRILL

1917. A PHYSIOLOGICAL STUDY OF THE CLIMATIC CONDITIONS OF MARYLAND AS MEASURED BY THE RATE OF PLANT GROWTH. Physiol. Res. 2: $341-405$.

HoFF, J. H. VAN'T

1884. ETUDES DE DYNAMIQUE CHIMIQUe. Amsterdam.

HOFFMAN, J.

1881. Comparative phenological Chart for central europe. Pettermann's Geog. Mitth.

Hoge, M. A.

1915. THE INFLUENCE OF TEMPERATURE UPON THE DEVELOPMENT OF A MENDELIAN CHARACTER. Jour. Expt. Zool. 18: 241-286.

HOPKINS, A. D.

1900. Hessian Fly IN West virginia. W. Va. Agr. Expt. Sta. Bul. 67: 242-243.

1918. GUIDES To AGRICUltURAL RESEARCH. Monthly Weather Review, U. S. Dept. Agr. Suppl. 9: 1-41. 
1919. THE BIOCliafatic LAw. Sci. Monthly. 8: 496-513.

HOWARD, L. O.

1896. SOME TEMPETATURE EFFECTS ON HOUSEHOLD INSECTS. U. S. Dept. Agr. Div. Ent. Bul. 6, n.s. p. 13-17.

Hunter, S. J. and GLENN, P. A.

1909. THE GREeN BUG AND its Natural ENEMIES. Univ. of Kans. Bul. 9: $165-200$.

HuNTER, W. D.

1908. A TENTATIVE LAW RELATING TO THE INCUBATION OF THE EGGS OF THE CATTLE TICK. Joux. Econ. Ent. 1: 51-55.

and Hinds, W. E.

1904. THE MEXiCAN COTTON BOLL WEevil. U. S. Dept. Agr. Bur. of Ent. Bul. 45 .

and HOOKER, W. A.

1907. THE NORTH AMERICAN Fever tiCk. U. S. Dept. Agr. Bur. of Ent. Bul. 72.

Huntington, E.

1915. Civilization and climate. New Haven.

JOHNSON, EARL S.

1923. MOISTURE RELATIONS OF PEACH BUDS DURING WINTER AND SPRING. Md. Agr. Expt. Sta. Bul 255.

Johannsen, A. C., and Krogh, A.

1914. THE INFLUENCE OF TEMPERATURE AND CERTAIN OTHER TACTORS ON THE GRowth of Fishes. Conseil Int. p. l'exploration de la mer. Publ. de circ. No. 68, Copenhagen.

KANITZ, ARISTIDES

1915. TEMPERATURE UND LEBENSVORGANGE. Berlin.

KING, HeLEN DEAN

1910. TEMPERATURE AS A F'ACTOR IN THE DETERMINATION OF SEX. Biol. Bul. 18: 131-137.

1903. EFFECT OF HEAT ON THE DEVELOPMENT OF TOAD EGgS. Biol. Bul 5 : 218-232.

INIGHT, HARRY H.

1924. ON THE NATURE OF THE COLOR PATTERNS IN HETEROPTERA WITH DATA ON THE EFFECTS PRODUCED BY TEMPERATURE AND Humidity. Ann. Ent. Soc. of Am. 17: 258-274.

KNOWLTON, HARRY E.

1922. STUdIES IN POLLEN WITH SPECIAL REFERENCE TO LONGEVITY. COYnell Agr. Expt. Sta. Memoir 52.

Knowlton, Frank P., and Starling, E. H.

1912. INFLUENCE OF TEMPERATURE ON THE ISOLATED MAMMALIAN HEART. Jour. Physiol. 44: 206-219.

IOPEC, STEFAN

1924. STUDIES ON THE INFLUENCE OF INANITION ON THE DEVELOPMENT AND DURATION OF LIFE IN INSECTS. Biol. Bul. 46: 1-23. 
KRAFKa, Joseph, JR.

1920. THE EFFECT OF TEMPERATURE UPON THE FACET-NUMBER IN THE BAR-EYED MUTANT OF DROSOPHILA. Jour. Gen. Physiol. 2: 409-464.

KRAMER, S. D.

1915. . EFFECT OF TEMPERATURE UPON THE LIFE CYCLE OF MUSCA DOMESTICA AND CUleX PIPIENS. Science, N. S. 41: 874-877.

Krogh, August

1914. ON THE RATE OF DEVELOPMENT AND CARBON DIOXIDE PRODUCTION OF CHRYSALIDES OF TENEBRIO MOLITOR AT DIFFERENT TEMPERATURES. Zeitschr. fur allgem. Physiol. 16: 178-190.

1916. THE RESPIRATORY EXCHANGE OF MAN AND ANIMALS London.

Larson, A. O., and Simmons, Perez

1923. NOTES ON THE BIOLOGY OF THE FOUR-SPOTTED BEAN WEEVIL, BRUCHUS QUADRIMACUlatUS FABR. Jour. Agr. Res. 22: 609616.

LEHENBAUER, P. A.

1914. THE GROWTH OF MAIZE SEEDLINGS IN RELATION TO TEMPERATURE. Physiol. Res. 1: 247-288.

LEPESCHKIN, S.

1912. LETHAL EFFECTS OF TEMPERATURE ON PLANT TISSUES. Bericht de, Deutsches Bot. Ges. 30 .

Lillie, R. F. and Knowlton, F. P.

1897. OF THE EFFECT OF TEMPERATURE UPON THE DEVELOPMENT OF ANIMALS. Zool. Bul. 1: 179-193.

LINSSER, CARL

1867. DIE PERIODISCHEN ERSCHEINUNGEN DES PFLANZEN-LEBENS IN IHREN VERHALTNISSE ZU DEN WARME-ERSCHEINUNG. Meb. Acad. Imp. St. Petersburg, (7) T. 11.

1869. UNTERSUCHUNGEN UEBER DIE PERIODISCHEN LEBENS-ERSCHEINUNGEN DER PFLANzen. Zweite AbhandIungen. Mem. Acad. Imp. St. Petersburg, (7) T. 13.

Livingstone, B. E.

1916. PHYSIOLOGICAL TEMPERATURE INDICES FOR THE STUDY OF PLANT GROWTH IN RELATION TO CLIMATIC CONDITIONS Physiol. Res. 1: $\quad 399-420$.

and Livingston, Grace J.

1913. TEMPERATURE COEFFICIENTS IN PLANT GEOGRAPHY AND CLIMATOLoGY. Bot. Gaz. 56: 349-376.

LOEB, JACQUES

1906. THE DYNAMICS OF LIVING MATTER. New York.

and NoRTHRUP, J. H.

1917. ON THE INFLUENCE OF FOOD AND TEMPERATURE UPON THE DURATION OF LIFE. Jour. Biol-Chem. 32: 103-121.

and WASTENEYS, H.

1911. ARE THE OXIDATIVE PROCESSES INDEPENDANT VARIABLES IN VITAL PRocesses. Biochem. Zr. 36: 345-356. 
INFLUENCE OF BASES IN THE DEVELOPMENTAL AND OXIDATIVE PROCESSES. Biochem. Zr. 37: 410-423. (Trans. titles).

MCLEAN, F. T.

1917. A PRELIMINARY STUDY OF CLIMATIC CONDITIONS IN MARYLAND AS Related to plant growth. Physiol. Res. 2: 129-208.

MCDOUGALL, D. T.

1907. FACTORS AFFECTING THE SEASONAL ACTIVITIES OF PLANTS. Plant World, 10: 217-237.

1914. AUTO-THERMAL INTEGRATIONS OF TEMPERATURE COMPLEXES. Am. Jour. Bot. 1: 186-193.

MELLOR, J. W.

1904. CHEMiCAL STATICS AND DYNAMics. Chap. 12 . London.

MERREIM, C. HART

1894. LAWS OF TEMPERATURE CONTROL OF THE GEOGRAPHIC DISTRIBUTION of terrestrial animals and Plants. Nat. Geog. Mag. 6: 228-238.

Morgan, T. H.

1907. EXPERIMENTAL ZOOLOGY. New York.

MORGULIS, S.

1924. EFFECTS OF ENVIRONMENTAL TEMPERATURE ON METABOLISM. Am. Jour. Phys. 71: 49-59.

NERNST, WALTER

1895. THEORETICAL CHEMISTRY. Chap. 4. (Translation by Palmer) London.

PeAirs, L. M.

1914. THE RELATION OF TEMPERATURE TO INSECT DEVELOPMENT. Jour. Ec. Ent. 7: 174-179.

Pierce, W. DWight

1916. A NEW INTERPRETATION OF THE RELATIONSHIPS OF TEMPERATURE AND HUMidity to insect DEVELOPMENT. Jour. Agr. Res. 5: 1183-1191.

Plough, HaRry H.

1917. THE EFFECT OF TEMPERATURE UPON CROSSING-OVER IN DROSOPHILA. Jour. Expt. Zool. 24: 147-209.

1921. FURTHER STUDIFS ON THE EFFECT OF TEMPERATURE ON CROSSINGover. Jour. Expt. Zool. 32: 187-201.

PRICE, H. L.

1910. THE APPLICATION OF METEOROLOGICAL DATA IN THE STUDY OF PHYSIOLOGICAL CONSTANTS. Ann. Rept. Virginia Agr. Expt. Sta. for 1909-1910.

1914. TEmperaturkoeffizienten. Zeitschr. for Allgem. Physiol. 16: 574-627. 
QUetelet, A.

1819. SUR LE CLIMAT DE LA BELGIQUE. Phenomenes periodiques des

Plants. Chap. 4. Annales de l'Observatoire. Bruxelles.

REAUMiUR, R. A. F. DE

1735. MEMIOIRS, ACAD. DES SCIENCE, p. 545. Paris.

REIBISCH, J.

1902. UEBER DEN EINFLUSS DER TEMPERATURE AUF DIE ENTWICKLUNG VON FISCHEIERN. Wissenchaft. Meeresuntersuch., N. F., Abt. Kiel, 6: 215-231.

Richardson, Chas. H.

1925. the oviposition Response of insects. U. S. Dept. Agr. Bul. 1324.

Roberts, Eliier

1918. FLUCTUATIONS IN A RECESSIVE MENDELIAN CHARACTER AND SELECTION. Jour. Expt. Zool. 27: 157-177.

ROGERS, ChAS. G.

1911. STUDIES UPON THE TEMPERATURE COEFFICIENT OF THE RATE OF HEART BEAT IN CERTAIN LIVING ANIMALS. Am. Jour. Physiol. 28: 81-93.

SANDERSON, E. DWIGHT

1908. THE INFLUCNCE OF TEMPERATURE IN THE NORTHERN DISTRIBUTION of INSECTS. Jour. Econ. Ent. 1: 245-262.

1908. THE RELATION OT TEMIPERATURE TO THE HIBERNATION OF INSECTS. Jour. Econ. Ent. 1: 56-65.

1910. THE RELATION OF TEMPERATURE TO THE GROWTH OF INSECTS. Jour. Econ. Ent. 3: 133-140.

Sanderson, E. Dwight, and Peairs, L. M.

1914. THE RELATION OF TEMPERATURE TO THE DEVELOPMENT OF INSECTS. N. Hamp. Agr. Expt. Sta. Tech. Bul. 7.

Schwetalla, Alphonse M.

1924. INFLUENCE OF TEMPERATURE ON RATE OF LOCOMOTION OF AMOEBA. Jour. Morph. 39: 465-514.

Severin, H. H. P. and Severin, H. C.

1910. THE EFFECT OF MIOISTURE AND DARKNESS ON THE EMERGENCE FROM THE EGG OF THE WALKING-STICK, DIAPHEROMERA FEMORATA, Say. Jour. Econ. Ent. 3: 479-481.

SHELFORD, V. E.

1917. PHYSIOLOGICAL PROBLEMS IN THE LIFE-HISTORIES OF ANIMALS WITH PARTICULAR REFERENCE TO THEIR SEASONAL APPEARANCE. Am. Nat. 52: 129-154.

1920. PHYSIOLOGICAL LIFE HISTORIES OF TERRESTRIAL ANIMALS AND MODERN METHODS OF REPRESENTING CLIMATE. Illinois Acad. of Science. 13 : 257-271. 
1926. AN EXPERIMENTAL INVESTIGATION OF THE RELATION OF THE CODLiNg moth to weather and Climate. Ill. Nat. Hist. Survey. Bul. 16, art. 5 .

SNYDER, CHAS. D.

1907. DER TEMERATUR KOEFFICIENT DER GESCHWINDIGKEIT DER NERVENLeitung. Arch. Anat. und Phys.-Physiol. Abt. Jahl. 1907: 113-145.

1908. A COMPARATIVE STUDY OF THE TEMPERATURE COEFFICIENTS OF VARIOUS PHYSIOLOGICAL ACtions. Am. Jour. Physiol. 22: 309334.

1911. ON THE MEANING OF THE VARIATION IN THE MAGNITUDE OF THE TEMPERATURE COEFFICIENT OF PHYSIOLOGICAL PROCESSES. Am. Jour. Physiol. 28: 167-175.

Stevens, NeIL E.

1917. INFLUENCE OF CLIMATIC FACTORS ON THE DEVELOPMENT OF ENDOTHIA PARASITICA. Am. Jour. Bot. $4: 1-32$.

1917. INFLUENCE OF TEMPERATURE ON THE DEVELOPMENT OF ENDOTHIA PARASITICA. Am. Jour. Bot. 4: 111-118.

1917. TEMPERATURES OF THE CRANBERRY REGIONS OF THE UNITED STATES in RELATION TO THE GROWTH OF CERTAIN FUNGi. Jour. Agr. Res. 11: 521-529.

SEYSTER, E. W.

1919. EYE-FACET NUMBER AS INFLUENCED BY TEMPERATURE IN BAR-EYED MUTANTS OF DROSOPhia. Biol. Bul. 37: 168-182.

SHREVE, FORREST

1914. THE ROLE OF WINTER TEMPERATURES IN DETERMINING THE DISTRIBUtion of Plants. Am. Jour. Bot. 1: 194-202.

Summers, Francis B.

1915. SOME STUDIES OF ENVIRON MENTAL INFLUENCE ON HEREDITY. Jour. Expt. Zool. 18.

TANGL, F.

1909. ZUR KENTNISS DES STOFF UND ENERGIEUMSATZES HOLOMETABOLER INSEKTEN WAHREND DER METAMORPHOSE. Pflugers Archiv. 13-: 1-55.

THOMAS, CYRUS

1879. RELATION OF TEMPERATURE TO THE DEVELOPMENT OF (LOCUST)

EGGS. Second. Ann. Rept. U. S. Ent. Comm. Chap. 5.

ГOTHILL, JOHN D.

1913. A STUDY OF THE VARIATION IN THE NORTH AMERICAN GREENBOTTLE FLIES. Ann. Ent. Soc. Am. VI: 241-256.

TOWER, W. L.

1912. MODIFICATION OF THE GERMINAL CONSTITUTION OF ORGANiSMS. Heredity and Eugenics, Chap. 7.

1918. THE MECHANISM OF EVOLUTION IN LEPTINOTARSA. Publication of the Carnegie Institute. 
Von Oettingen, A. J.

1879. PHANOLOGIE DER DORPATER LIGNOSEN. Archiv fur die Naturkunde Liv-, Ehst- und Kurlands. Zweite Serie. pp. 241352. Bande VIII. Dritte Lieferung. Dorpat.

VERWORN, MAX

1899. General Physiology. (Trans. by F. S. Lee) London.

Wallich, Claudius

1900. Dept. U. S. Fish. Comm. for 1900, Pt. 26. House Documents 122, 56th Congress.

WAUGH, F. A.

1898.

A CONTRIBUTION TO THE KNOWLEDGE OF PHYSIOLOGICAL CONSTANTS. Ann. Rept. Vt. Agr. Expt. Sta. for 1897-98: 263272.

WARDER, R. B.

1881. In Am. Chem. Jour. 3: 203.

WEINLAND, E.

1906. PUPPEN VON CALLIPHORA. Ziet. 4. Biol. 48, 87-140. Muenschen u. Berlin.

Zeleny, Charles

1920. In Jour. Expt. Zool. 30: 293:324.

1920. Tabulation of factorial values. Am. Nat. 54: 358-362.

1922. In Genetics $7: 1-115$. 


\section{.}





\section{HECKMAN}

BINDERY INC.

\section{JUNE 99}


